\title{
A Au(I)-Precatalyst with a Cyclopropenium Counterion: An Unusual
}

\section{Ion Pair}

\author{
Roya Mir, Travis Dudding*
}

Department of Chemistry, Brock University, 1812 Sir Isaac Brock Way, St. Catharines, Ontario, Canada, L2S 3A1.

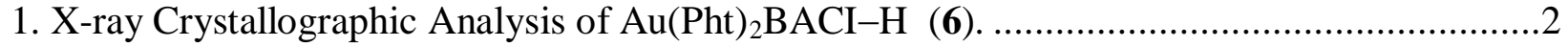

2. DFT calculated geometries and thermochemical data. …......................................................15

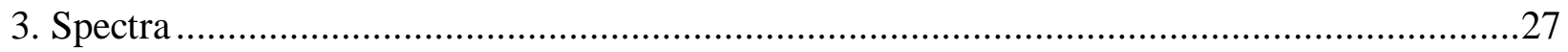




\section{X-ray Crystallographic Analysis of $\mathrm{Au}(\mathrm{Pht})_{2} \mathrm{BACI}-\mathrm{H}$ (6).}

Identification code

Empirical formula

Formula weight

Temperature

Wavelength

Crystal system

Space group

Unit cell dimensions

Volume

$\mathrm{Z}$

Density (calculated)

Absorption coefficient

$\mathrm{F}(000)$

Crystal size

Theta range for data collection

Index ranges

Reflections collected

Independent reflections

Completeness to theta $=25.242^{\circ}$

Absorption correction

Max. and min. transmission

Refinement method

Data / restraints / parameters

Goodness-of-fit on $\mathrm{F}^{2}$

Final R indices [I $>2 \operatorname{sigma}(\mathrm{I})]$

$\mathrm{R}$ indices (all data)

Extinction coefficient

Largest diff. peak and hole
RM08729b_0m_a

C31 H37 Au N4 O4

726.61

173(2) K

$0.71073 \AA$

Monoclinic

$\mathrm{C} 2 / \mathrm{c}$

$\mathrm{a}=17.7379(5) \AA \quad \alpha=90^{\circ}$.

$\mathrm{b}=9.4418(3) \AA \quad \beta=106.1382(15)^{\circ}$.

$\mathrm{c}=18.8452(6) \AA \quad \gamma=90^{\circ}$.

3031.78(16) $\AA^{3}$

4

$1.592 \mathrm{Mg} / \mathrm{m}^{3}$

$4.893 \mathrm{~mm}^{-1}$

1448

$0.453 \times 0.236 \times 0.126 \mathrm{~mm}^{3}$

2.250 to $33.171^{\circ}$.

$-27<=\mathrm{h}<=27,-14<=\mathrm{k}<=14,-28<=1<=28$

42033

$5803[\mathrm{R}(\mathrm{int})=0.0363]$

$100.0 \%$

Numerical

0.4046 and 0.1321

Full-matrix least-squares on $\mathrm{F}^{2}$

$5803 / 0 / 217$

1.046

$\mathrm{R} 1=0.0303, \mathrm{wR} 2=0.0758$

$\mathrm{R} 1=0.0429, \mathrm{wR} 2=0.0827$

$\mathrm{n} / \mathrm{a}$

1.502 and -1.202 e. $\AA^{-3}$ 
Table 2. Atomic coordinates ( $\times 10^{4}$ ) and equivalent isotropic displacement parameters $\left(\AA^{2} \times 10^{3}\right)$ for rm08729b_0m_a. U(eq) is defined as one third of the trace of the orthogonalized $U^{i j}$ tensor.

\begin{tabular}{|c|c|c|c|c|}
\hline & $\mathrm{x}$ & $\mathrm{y}$ & $\mathrm{z}$ & $\mathrm{U}(\mathrm{eq})$ \\
\hline $\mathrm{Au}(1)$ & 2500 & 7500 & 0 & $32(1)$ \\
\hline $\mathrm{O}(1)$ & $3108(2)$ & $4966(4)$ & $1267(2)$ & $80(1)$ \\
\hline $\mathrm{O}(2)$ & $1117(2)$ & $8173(3)$ & $799(2)$ & $55(1)$ \\
\hline $\mathrm{N}(1)$ & $2178(1)$ & $6712(2)$ & $858(1)$ & $31(1)$ \\
\hline $\mathrm{C}(1)$ & 2534(2) & $5622(3)$ & $1316(2)$ & $42(1)$ \\
\hline $\mathrm{C}(2)$ & 2102(2) & $5412(3)$ & 1882(2) & $36(1)$ \\
\hline$C(3)$ & $1504(2)$ & $6382(3)$ & 1741(1) & $30(1)$ \\
\hline $\mathrm{C}(4)$ & 1551(2) & $7220(3)$ & 1082(2) & $30(1)$ \\
\hline$C(5)$ & $1007(2)$ & $6485(4)$ & $2184(2)$ & $48(1)$ \\
\hline$C(6)$ & $1132(3)$ & $5559(5)$ & 2781(2) & $59(1)$ \\
\hline $\mathrm{C}(7)$ & $1727(3)$ & $4575(5)$ & 2916(2) & $63(1)$ \\
\hline$C(8)$ & $2223(2)$ & $4475(4)$ & $2467(2)$ & $58(1)$ \\
\hline $\mathrm{C}(1 \mathrm{~A})$ & $385(3)$ & 10721(5) & $-44(3)$ & $26(1)$ \\
\hline$C(3 A)$ & $-19(3)$ & $9593(5)$ & $-461(3)$ & $27(1)$ \\
\hline $\mathrm{C}(2 \mathrm{~A})$ & $591(3)$ & $10223(6)$ & $-645(3)$ & $30(1)$ \\
\hline $\mathrm{N}(1 \mathrm{~A})$ & $505(2)$ & $11609(3)$ & $506(2)$ & $35(1)$ \\
\hline $\mathrm{N}(2 \mathrm{~A})$ & $-574(2)$ & $8654(3)$ & $-603(2)$ & $35(1)$ \\
\hline $\mathrm{C}(4 \mathrm{~A})$ & $1186(2)$ & $12590(3)$ & $604(3)$ & $52(2)$ \\
\hline$C(5 A)$ & $1928(2)$ & 11814(4) & $637(3)$ & $62(2)$ \\
\hline$C(6 A)$ & $993(3)$ & 13710(3) & $-7(3)$ & $90(3)$ \\
\hline $\mathrm{C}(7 \mathrm{~A})$ & $74(2)$ & 11585(4) & 1063(2) & $78(3)$ \\
\hline $\mathrm{C}(8 \mathrm{~A})$ & $-360(3)$ & $12970(4)$ & 1066(3) & $99(2)$ \\
\hline $\mathrm{C}(9 \mathrm{~A})$ & $619(3)$ & $11229(4)$ & $1815(2)$ & $102(4)$ \\
\hline$C(10 A)$ & $-1092(2)$ & $8367(3)$ & $-123(2)$ & $52(2)$ \\
\hline$C(11 \mathrm{~A})$ & $-1955(2)$ & $8754(4)$ & $-568(3)$ & $62(2)$ \\
\hline$C(12 A)$ & $-1035(3)$ & $6866(3)$ & $153(3)$ & $90(3)$ \\
\hline$C(13 A)$ & $-615(3)$ & $7689(4)$ & $-1253(3)$ & $71(3)$ \\
\hline$C(14 A)$ & $-906(3)$ & $8450(5)$ & $-1980(2)$ & $102(4)$ \\
\hline$C(15 A)$ & $185(3)$ & $7005(4)$ & $-1165(3)$ & $99(2)$ \\
\hline
\end{tabular}


Table 3. Bond lengths $[\AA ̊]$ and angles $\left[{ }^{\circ}\right]$ for rm08729b_0m_a.

\begin{tabular}{|c|c|}
\hline $\mathrm{Au}(1)-\mathrm{N}(1) \# 1$ & $2.002(2)$ \\
\hline $\mathrm{Au}(1)-\mathrm{N}(1)$ & $2.003(2)$ \\
\hline $\mathrm{O}(1)-\mathrm{C}(1)$ & $1.217(4)$ \\
\hline $\mathrm{O}(2)-\mathrm{C}(4)$ & $1.208(3)$ \\
\hline $\mathrm{N}(1)-\mathrm{C}(1)$ & $1.379(4)$ \\
\hline $\mathrm{N}(1)-\mathrm{C}(4)$ & $1.380(3)$ \\
\hline $\mathrm{C}(1)-\mathrm{C}(2)$ & $1.489(5)$ \\
\hline $\mathrm{C}(2)-\mathrm{C}(3)$ & $1.370(4)$ \\
\hline $\mathrm{C}(2)-\mathrm{C}(8)$ & $1.383(4)$ \\
\hline$C(3)-C(5)$ & $1.378(4)$ \\
\hline $\mathrm{C}(3)-\mathrm{C}(4)$ & $1.493(4)$ \\
\hline$C(5)-C(6)$ & $1.393(5)$ \\
\hline $\mathrm{C}(5)-\mathrm{H}(5)$ & $0.92(6)$ \\
\hline$C(6)-C(7)$ & $1.374(6)$ \\
\hline $\mathrm{C}(6)-\mathrm{H}(6)$ & $0.90(4)$ \\
\hline $\mathrm{C}(7)-\mathrm{C}(8)$ & $1.384(6)$ \\
\hline $\mathrm{C}(7)-\mathrm{H}(7)$ & $0.89(5)$ \\
\hline $\mathrm{C}(8)-\mathrm{H}(8)$ & $0.99(5)$ \\
\hline $\mathrm{C}(1 \mathrm{~A})-\mathrm{N}(1 \mathrm{~A})$ & $1.304(6)$ \\
\hline $\mathrm{C}(1 \mathrm{~A})-\mathrm{C}(2 \mathrm{~A})$ & $1.368(7)$ \\
\hline $\mathrm{C}(1 \mathrm{~A})-\mathrm{C}(3 \mathrm{~A})$ & 1.397(7) \\
\hline $\mathrm{C}(3 \mathrm{~A})-\mathrm{N}(2 \mathrm{~A})$ & 1.297(6) \\
\hline $\mathrm{C}(3 \mathrm{~A})-\mathrm{C}(2 \mathrm{~A})$ & $1.362(7)$ \\
\hline $\mathrm{C}(2 \mathrm{~A})-\mathrm{H}(2 \mathrm{~A})$ & 0.9500 \\
\hline $\mathrm{N}(1 \mathrm{~A})-\mathrm{C}(7 \mathrm{~A})$ & 1.4603 \\
\hline $\mathrm{N}(1 \mathrm{~A})-\mathrm{C}(4 \mathrm{~A})$ & 1.4916 \\
\hline $\mathrm{N}(2 \mathrm{~A})-\mathrm{C}(10 \mathrm{~A})$ & 1.4816 \\
\hline $\mathrm{N}(2 \mathrm{~A})-\mathrm{C}(13 \mathrm{~A})$ & 1.5124 \\
\hline $\mathrm{C}(4 \mathrm{~A})-\mathrm{C}(5 \mathrm{~A})$ & 1.4927 \\
\hline $\mathrm{C}(4 \mathrm{~A})-\mathrm{C}(6 \mathrm{~A})$ & 1.5309 \\
\hline $\mathrm{C}(4 \mathrm{~A})-\mathrm{H}(4 \mathrm{~A})$ & 1.0000 \\
\hline $\mathrm{C}(5 \mathrm{~A})-\mathrm{H}(5 \mathrm{C})$ & 0.9800 \\
\hline $\mathrm{C}(5 \mathrm{~A})-\mathrm{H}(5 \mathrm{~B})$ & 0.9800 \\
\hline $\mathrm{C}(5 \mathrm{~A})-\mathrm{H}(5 \mathrm{~A})$ & 0.9800 \\
\hline $\mathrm{C}(6 \mathrm{~A})-\mathrm{H}(6 \mathrm{C})$ & 0.9800 \\
\hline
\end{tabular}




\begin{tabular}{|c|c|}
\hline $\mathrm{C}(6 \mathrm{~A})-\mathrm{H}(6 \mathrm{~B})$ & 0.9800 \\
\hline $\mathrm{C}(6 \mathrm{~A})-\mathrm{H}(6 \mathrm{~A})$ & 0.9800 \\
\hline $\mathrm{C}(7 \mathrm{~A})-\mathrm{C}(9 \mathrm{~A})$ & 1.5142 \\
\hline $\mathrm{C}(7 \mathrm{~A})-\mathrm{C}(8 \mathrm{~A})$ & 1.5181 \\
\hline $\mathrm{C}(7 \mathrm{~A})-\mathrm{H}(7 \mathrm{~A})$ & 1.0000 \\
\hline $\mathrm{C}(8 \mathrm{~A})-\mathrm{H}(8 \mathrm{C})$ & 0.9800 \\
\hline $\mathrm{C}(8 \mathrm{~A})-\mathrm{H}(8 \mathrm{~B})$ & 0.9800 \\
\hline $\mathrm{C}(8 \mathrm{~A})-\mathrm{H}(8 \mathrm{~A})$ & 0.9800 \\
\hline $\mathrm{C}(9 \mathrm{~A})-\mathrm{H}(9 \mathrm{C})$ & 0.9800 \\
\hline $\mathrm{C}(9 \mathrm{~A})-\mathrm{H}(9 \mathrm{~B})$ & 0.9800 \\
\hline $\mathrm{C}(9 \mathrm{~A})-\mathrm{H}(9 \mathrm{~A})$ & 0.9800 \\
\hline$C(10 A)-C(12 A)$ & 1.5036 \\
\hline$C(10 A)-C(11 A)$ & 1.5705 \\
\hline $\mathrm{C}(10 \mathrm{~A})-\mathrm{H}(10 \mathrm{~A})$ & 1.0000 \\
\hline $\mathrm{C}(11 \mathrm{~A})-\mathrm{H}(11 \mathrm{C})$ & 0.9800 \\
\hline $\mathrm{C}(11 \mathrm{~A})-\mathrm{H}(11 \mathrm{~B})$ & 0.9800 \\
\hline $\mathrm{C}(11 \mathrm{~A})-\mathrm{H}(11 \mathrm{~A})$ & 0.9800 \\
\hline $\mathrm{C}(12 \mathrm{~A})-\mathrm{H}(12 \mathrm{C})$ & 0.9800 \\
\hline $\mathrm{C}(12 \mathrm{~A})-\mathrm{H}(12 \mathrm{~B})$ & 0.9800 \\
\hline $\mathrm{C}(12 \mathrm{~A})-\mathrm{H}(12 \mathrm{~A})$ & 0.9800 \\
\hline$C(13 A)-C(14 A)$ & 1.5045 \\
\hline$C(13 A)-C(15 A)$ & 1.5253 \\
\hline $\mathrm{C}(13 \mathrm{~A})-\mathrm{H}(13 \mathrm{~A})$ & 1.0000 \\
\hline $\mathrm{C}(14 \mathrm{~A})-\mathrm{H}(14 \mathrm{C})$ & 0.9800 \\
\hline $\mathrm{C}(14 \mathrm{~A})-\mathrm{H}(14 \mathrm{~B})$ & 0.9800 \\
\hline $\mathrm{C}(14 \mathrm{~A})-\mathrm{H}(14 \mathrm{~A})$ & 0.9800 \\
\hline $\mathrm{C}(15 \mathrm{~A})-\mathrm{H}(15 \mathrm{C})$ & 0.9800 \\
\hline $\mathrm{C}(15 \mathrm{~A})-\mathrm{H}(15 \mathrm{~B})$ & 0.9800 \\
\hline $\mathrm{C}(15 \mathrm{~A})-\mathrm{H}(15 \mathrm{~A})$ & 0.9800 \\
\hline $\mathrm{N}(1) \# 1-\mathrm{Au}(1)-\mathrm{N}(1)$ & 180.0 \\
\hline $\mathrm{C}(1)-\mathrm{N}(1)-\mathrm{C}(4)$ & $109.6(2)$ \\
\hline $\mathrm{C}(1)-\mathrm{N}(1)-\mathrm{Au}(1)$ & $126.77(19)$ \\
\hline $\mathrm{C}(4)-\mathrm{N}(1)-\mathrm{Au}(1)$ & $123.58(18)$ \\
\hline $\mathrm{O}(1)-\mathrm{C}(1)-\mathrm{N}(1)$ & $125.5(3)$ \\
\hline $\mathrm{O}(1)-\mathrm{C}(1)-\mathrm{C}(2)$ & $126.5(3)$ \\
\hline $\mathrm{N}(1)-\mathrm{C}(1)-\mathrm{C}(2)$ & $108.1(2)$ \\
\hline
\end{tabular}




\begin{tabular}{|c|c|}
\hline$C(3)-C(2)-C(8)$ & $121.5(3)$ \\
\hline $\mathrm{C}(3)-\mathrm{C}(2)-\mathrm{C}(1)$ & $107.2(2)$ \\
\hline $\mathrm{C}(8)-\mathrm{C}(2)-\mathrm{C}(1)$ & $131.3(3)$ \\
\hline$C(2)-C(3)-C(5)$ & $121.4(3)$ \\
\hline $\mathrm{C}(2)-\mathrm{C}(3)-\mathrm{C}(4)$ & $107.3(2)$ \\
\hline$C(5)-C(3)-C(4)$ & $131.2(3)$ \\
\hline $\mathrm{O}(2)-\mathrm{C}(4)-\mathrm{N}(1)$ & $126.3(3)$ \\
\hline $\mathrm{O}(2)-\mathrm{C}(4)-\mathrm{C}(3)$ & $125.9(3)$ \\
\hline $\mathrm{N}(1)-\mathrm{C}(4)-\mathrm{C}(3)$ & $107.8(2)$ \\
\hline$C(3)-C(5)-C(6)$ & $117.4(4)$ \\
\hline $\mathrm{C}(3)-\mathrm{C}(5)-\mathrm{H}(5)$ & $119(4)$ \\
\hline $\mathrm{C}(6)-\mathrm{C}(5)-\mathrm{H}(5)$ & $124(4)$ \\
\hline$C(7)-C(6)-C(5)$ & $121.1(4)$ \\
\hline $\mathrm{C}(7)-\mathrm{C}(6)-\mathrm{H}(6)$ & $116(3)$ \\
\hline $\mathrm{C}(5)-\mathrm{C}(6)-\mathrm{H}(6)$ & $123(3)$ \\
\hline $\mathrm{C}(6)-\mathrm{C}(7)-\mathrm{C}(8)$ & $121.2(3)$ \\
\hline $\mathrm{C}(6)-\mathrm{C}(7)-\mathrm{H}(7)$ & $121(3)$ \\
\hline $\mathrm{C}(8)-\mathrm{C}(7)-\mathrm{H}(7)$ & $118(3)$ \\
\hline$C(7)-C(8)-C(2)$ & $117.4(4)$ \\
\hline $\mathrm{C}(7)-\mathrm{C}(8)-\mathrm{H}(8)$ & $126(3)$ \\
\hline $\mathrm{C}(2)-\mathrm{C}(8)-\mathrm{H}(8)$ & $117(3)$ \\
\hline $\mathrm{N}(1 \mathrm{~A})-\mathrm{C}(1 \mathrm{~A})-\mathrm{C}(2 \mathrm{~A})$ & $149.0(5)$ \\
\hline $\mathrm{N}(1 \mathrm{~A})-\mathrm{C}(1 \mathrm{~A})-\mathrm{C}(3 \mathrm{~A})$ & $151.9(5)$ \\
\hline$C(2 A)-C(1 A)-C(3 A)$ & $59.0(4)$ \\
\hline $\mathrm{N}(2 \mathrm{~A})-\mathrm{C}(3 \mathrm{~A})-\mathrm{C}(2 \mathrm{~A})$ & $149.0(5)$ \\
\hline $\mathrm{N}(2 \mathrm{~A})-\mathrm{C}(3 \mathrm{~A})-\mathrm{C}(1 \mathrm{~A})$ & $151.5(5)$ \\
\hline$C(2 A)-C(3 A)-C(1 A)$ & $59.4(4)$ \\
\hline$C(3 A)-C(2 A)-C(1 A)$ & $61.5(4)$ \\
\hline $\mathrm{C}(3 \mathrm{~A})-\mathrm{C}(2 \mathrm{~A})-\mathrm{H}(2 \mathrm{~A})$ & 149.2 \\
\hline $\mathrm{C}(1 \mathrm{~A})-\mathrm{C}(2 \mathrm{~A})-\mathrm{H}(2 \mathrm{~A})$ & 149.2 \\
\hline $\mathrm{C}(1 \mathrm{~A})-\mathrm{N}(1 \mathrm{~A})-\mathrm{C}(7 \mathrm{~A})$ & $123.9(2)$ \\
\hline $\mathrm{C}(1 \mathrm{~A})-\mathrm{N}(1 \mathrm{~A})-\mathrm{C}(4 \mathrm{~A})$ & $116.5(3)$ \\
\hline $\mathrm{C}(7 \mathrm{~A})-\mathrm{N}(1 \mathrm{~A})-\mathrm{C}(4 \mathrm{~A})$ & 119.2 \\
\hline $\mathrm{C}(3 \mathrm{~A})-\mathrm{N}(2 \mathrm{~A})-\mathrm{C}(10 \mathrm{~A})$ & $124.2(3)$ \\
\hline $\mathrm{C}(3 \mathrm{~A})-\mathrm{N}(2 \mathrm{~A})-\mathrm{C}(13 \mathrm{~A})$ & $116.3(3)$ \\
\hline $\mathrm{C}(10 \mathrm{~A})-\mathrm{N}(2 \mathrm{~A})-\mathrm{C}(13 \mathrm{~A})$ & 119.1 \\
\hline $\mathrm{N}(1 \mathrm{~A})-\mathrm{C}(4 \mathrm{~A})-\mathrm{C}(5 \mathrm{~A})$ & 112.0 \\
\hline
\end{tabular}




\begin{tabular}{|c|c|}
\hline $\mathrm{N}(1 \mathrm{~A})-\mathrm{C}(4 \mathrm{~A})-\mathrm{C}(6 \mathrm{~A})$ & 109. \\
\hline$C(5 A)-C(4 A)-C(6 A)$ & 1 \\
\hline $\mathrm{N}(1 \mathrm{~A})-\mathrm{C}(4 \mathrm{~A})-\mathrm{H}(4 \mathrm{~A})$ & 107 \\
\hline $\mathrm{C}(5 \mathrm{~A})-\mathrm{C}(4 \mathrm{~A})-\mathrm{H}(4 \mathrm{~A})$ & 107 \\
\hline $\mathrm{C}(6 \mathrm{~A})-\mathrm{C}(4 \mathrm{~A})-\mathrm{H}(4 \mathrm{~A})$ & 1 \\
\hline $\mathrm{C}(4 \mathrm{~A})-\mathrm{C}(5 \mathrm{~A})-\mathrm{H}(5 \mathrm{C})$ & 109 \\
\hline $\mathrm{C}(4 \mathrm{~A})-\mathrm{C}(5 \mathrm{~A})-\mathrm{H}(5 \mathrm{~B})$ & 109 \\
\hline $\mathrm{H}(5 \mathrm{C})-\mathrm{C}(5 \mathrm{~A})-\mathrm{H}(5 \mathrm{~B})$ & 109 \\
\hline $\mathrm{C}(4 \mathrm{~A})-\mathrm{C}(5 \mathrm{~A})-\mathrm{H}(5 \mathrm{~A})$ & 109 \\
\hline $\mathrm{H}(5 \mathrm{C})-\mathrm{C}(5 \mathrm{~A})-\mathrm{H}(5 \mathrm{~A})$ & 109 \\
\hline $\mathrm{H}(5 \mathrm{~B})-\mathrm{C}(5 \mathrm{~A})-\mathrm{H}(5 \mathrm{~A})$ & 109 \\
\hline $\mathrm{C}(4 \mathrm{~A})-\mathrm{C}(6 \mathrm{~A})-\mathrm{H}(6 \mathrm{C})$ & 100 \\
\hline$C(4 A)-C(6 A)-H(6 B)$ & 109 \\
\hline $\mathrm{H}(6 \mathrm{C})-\mathrm{C}(6 \mathrm{~A})-\mathrm{H}(6 \mathrm{~B})$ & 109 \\
\hline$C(4 A)-C(6 A)-H(6 A)$ & 109 \\
\hline $\mathrm{H}(6 \mathrm{C})-\mathrm{C}(6 \mathrm{~A})-\mathrm{H}(6 \mathrm{~A})$ & 10 \\
\hline $\mathrm{H}(6 \mathrm{~B})-\mathrm{C}(6 \mathrm{~A})-\mathrm{H}(6 \mathrm{~A})$ & 109 \\
\hline $\mathrm{N}(1 \mathrm{~A})-\mathrm{C}(7 \mathrm{~A})-\mathrm{C}(9 \mathrm{~A})$ & 110. \\
\hline $\mathrm{N}(1 \mathrm{~A})-\mathrm{C}(7 \mathrm{~A})-\mathrm{C}(8 \mathrm{~A})$ & 110 \\
\hline $\mathrm{C}(9 \mathrm{~A})-\mathrm{C}(7 \mathrm{~A})-\mathrm{C}(8 \mathrm{~A})$ & 112 \\
\hline $\mathrm{N}(1 \mathrm{~A})-\mathrm{C}(7 \mathrm{~A})-\mathrm{H}(7 \mathrm{~A})$ & to \\
\hline $\mathrm{C}(9 \mathrm{~A})-\mathrm{C}(7 \mathrm{~A})-\mathrm{H}(7 \mathrm{~A})$ & 107 \\
\hline $\mathrm{C}(8 \mathrm{~A})-\mathrm{C}(7 \mathrm{~A})-\mathrm{H}(7 \mathrm{~A})$ & 107 \\
\hline $\mathrm{C}(7 \mathrm{~A})-\mathrm{C}(8 \mathrm{~A})-\mathrm{H}(8 \mathrm{C})$ & 100 \\
\hline $\mathrm{C}(7 \mathrm{~A})-\mathrm{C}(8 \mathrm{~A})-\mathrm{H}(8 \mathrm{~B})$ & 109 \\
\hline $\mathrm{H}(8 \mathrm{C})-\mathrm{C}(8 \mathrm{~A})-\mathrm{H}(8 \mathrm{~B})$ & 109 \\
\hline $\mathrm{C}(7 \mathrm{~A})-\mathrm{C}(8 \mathrm{~A})-\mathrm{H}(8 \mathrm{~A})$ & 10 \\
\hline $\mathrm{H}(8 \mathrm{C})-\mathrm{C}(8 \mathrm{~A})-\mathrm{H}(8 \mathrm{~A})$ & 109 \\
\hline $\mathrm{H}(8 \mathrm{~B})-\mathrm{C}(8 \mathrm{~A})-\mathrm{H}(8 \mathrm{~A})$ & 10 \\
\hline $\mathrm{C}(7 \mathrm{~A})-\mathrm{C}(9 \mathrm{~A})-\mathrm{H}(9 \mathrm{C})$ & 10 \\
\hline $\mathrm{C}(7 \mathrm{~A})-\mathrm{C}(9 \mathrm{~A})-\mathrm{H}(9 \mathrm{~B})$ & 10 \\
\hline $\mathrm{H}(9 \mathrm{C})-\mathrm{C}(9 \mathrm{~A})-\mathrm{H}(9 \mathrm{~B})$ & 109. \\
\hline $\mathrm{C}(7 \mathrm{~A})-\mathrm{C}(9 \mathrm{~A})-\mathrm{H}(9 \mathrm{~A})$ & 10 \\
\hline $\mathrm{H}(9 \mathrm{C})-\mathrm{C}(9 \mathrm{~A})-\mathrm{H}(9 \mathrm{~A})$ & 10 \\
\hline $\mathrm{H}(9 \mathrm{~B})-\mathrm{C}(9 \mathrm{~A})-\mathrm{H}(9 \mathrm{~A})$ & 10 \\
\hline $\mathrm{N}(2 \mathrm{~A})-\mathrm{C}(10 \mathrm{~A})-\mathrm{C}(12 \mathrm{~A})$ & 11 \\
\hline$N(2 A)-C(10 A)-C(11 A)$ & \\
\hline
\end{tabular}




\begin{tabular}{|c|c|}
\hline$C(12 A)-C(10 A)-C(11 A)$ & 111.4 \\
\hline $\mathrm{N}(2 \mathrm{~A})-\mathrm{C}(10 \mathrm{~A})-\mathrm{H}(10 \mathrm{~A})$ & 108.1 \\
\hline$C(12 A)-C(10 A)-H(10 A)$ & 108.1 \\
\hline$C(11 A)-C(10 A)-H(10 A)$ & 108.1 \\
\hline $\mathrm{C}(10 \mathrm{~A})-\mathrm{C}(11 \mathrm{~A})-\mathrm{H}(11 \mathrm{C})$ & 109.5 \\
\hline $\mathrm{C}(10 \mathrm{~A})-\mathrm{C}(11 \mathrm{~A})-\mathrm{H}(11 \mathrm{~B})$ & 109.5 \\
\hline $\mathrm{H}(11 \mathrm{C})-\mathrm{C}(11 \mathrm{~A})-\mathrm{H}(11 \mathrm{~B})$ & 109.5 \\
\hline $\mathrm{C}(10 \mathrm{~A})-\mathrm{C}(11 \mathrm{~A})-\mathrm{H}(11 \mathrm{~A})$ & 109.5 \\
\hline $\mathrm{H}(11 \mathrm{C})-\mathrm{C}(11 \mathrm{~A})-\mathrm{H}(11 \mathrm{~A})$ & 109.5 \\
\hline$H(11 B)-C(11 A)-H(11 A)$ & 109.5 \\
\hline $\mathrm{C}(10 \mathrm{~A})-\mathrm{C}(12 \mathrm{~A})-\mathrm{H}(12 \mathrm{C})$ & 109.5 \\
\hline $\mathrm{C}(10 \mathrm{~A})-\mathrm{C}(12 \mathrm{~A})-\mathrm{H}(12 \mathrm{~B})$ & 109.5 \\
\hline $\mathrm{H}(12 \mathrm{C})-\mathrm{C}(12 \mathrm{~A})-\mathrm{H}(12 \mathrm{~B})$ & 109.5 \\
\hline$C(10 A)-C(12 A)-H(12 A)$ & 109.5 \\
\hline $\mathrm{H}(12 \mathrm{C})-\mathrm{C}(12 \mathrm{~A})-\mathrm{H}(12 \mathrm{~A})$ & 109.5 \\
\hline $\mathrm{H}(12 \mathrm{~B})-\mathrm{C}(12 \mathrm{~A})-\mathrm{H}(12 \mathrm{~A})$ & 109.5 \\
\hline $\mathrm{C}(14 \mathrm{~A})-\mathrm{C}(13 \mathrm{~A})-\mathrm{N}(2 \mathrm{~A})$ & 112.1 \\
\hline$C(14 A)-C(13 A)-C(15 A)$ & 112.0 \\
\hline $\mathrm{N}(2 \mathrm{~A})-\mathrm{C}(13 \mathrm{~A})-\mathrm{C}(15 \mathrm{~A})$ & 109.5 \\
\hline $\mathrm{C}(14 \mathrm{~A})-\mathrm{C}(13 \mathrm{~A})-\mathrm{H}(13 \mathrm{~A})$ & 107.7 \\
\hline $\mathrm{N}(2 \mathrm{~A})-\mathrm{C}(13 \mathrm{~A})-\mathrm{H}(13 \mathrm{~A})$ & 107.7 \\
\hline$C(15 A)-C(13 A)-H(13 A)$ & 107.7 \\
\hline$C(13 A)-C(14 A)-H(14 C)$ & 109.5 \\
\hline $\mathrm{C}(13 \mathrm{~A})-\mathrm{C}(14 \mathrm{~A})-\mathrm{H}(14 \mathrm{~B})$ & 109.5 \\
\hline $\mathrm{H}(14 \mathrm{C})-\mathrm{C}(14 \mathrm{~A})-\mathrm{H}(14 \mathrm{~B})$ & 109.5 \\
\hline $\mathrm{C}(13 \mathrm{~A})-\mathrm{C}(14 \mathrm{~A})-\mathrm{H}(14 \mathrm{~A})$ & 109.5 \\
\hline $\mathrm{H}(14 \mathrm{C})-\mathrm{C}(14 \mathrm{~A})-\mathrm{H}(14 \mathrm{~A})$ & 109.5 \\
\hline $\mathrm{H}(14 \mathrm{~B})-\mathrm{C}(14 \mathrm{~A})-\mathrm{H}(14 \mathrm{~A})$ & 109.5 \\
\hline$C(13 A)-C(15 A)-H(15 C)$ & 109.5 \\
\hline$C(13 A)-C(15 A)-H(15 B)$ & 109.5 \\
\hline $\mathrm{H}(15 \mathrm{C})-\mathrm{C}(15 \mathrm{~A})-\mathrm{H}(15 \mathrm{~B})$ & 109.5 \\
\hline$C(13 A)-C(15 A)-H(15 A)$ & 109.5 \\
\hline $\mathrm{H}(15 \mathrm{C})-\mathrm{C}(15 \mathrm{~A})-\mathrm{H}(15 \mathrm{~A})$ & 109.5 \\
\hline $\mathrm{H}(15 \mathrm{~B})-\mathrm{C}(15 \mathrm{~A})-\mathrm{H}(15 \mathrm{~A})$ & 109.5 \\
\hline
\end{tabular}

Symmetry transformations used to generate equivalent atoms:

$\# 1-x+1 / 2,-y+3 / 2,-z$ 
Table 4. Anisotropic displacement parameters $\left(\AA^{2} \times 10^{3}\right)$ for rm08729b_0m_a. The anisotropic displacement factor exponent takes the form: $-2 \pi^{2}\left[h^{2} a^{* 2} U^{11}+\ldots+2 h k a^{*} b^{*} U^{12}\right]$

\begin{tabular}{|c|c|c|c|c|c|c|}
\hline & $\mathrm{U}^{11}$ & $\mathrm{U}^{22}$ & $\mathrm{U}^{33}$ & $\mathrm{U}^{23}$ & $\mathrm{U}^{13}$ & $\mathrm{U}^{12}$ \\
\hline $\mathrm{Au}(1)$ & $26(1)$ & $33(1)$ & $41(1)$ & $-6(1)$ & $16(1)$ & $-5(1)$ \\
\hline $\mathrm{O}(1)$ & $65(2)$ & $83(2)$ & $99(2)$ & $9(2)$ & $33(2)$ & $45(2)$ \\
\hline $\mathrm{O}(2)$ & $50(1)$ & $55(2)$ & $71(2)$ & $36(1)$ & $35(1)$ & $26(1)$ \\
\hline $\mathrm{N}(1)$ & $26(1)$ & $30(1)$ & $38(1)$ & $-2(1)$ & $12(1)$ & $0(1)$ \\
\hline $\mathrm{C}(1)$ & $32(1)$ & $39(1)$ & $54(2)$ & $-1(1)$ & $9(1)$ & $9(1)$ \\
\hline$C(2)$ & $32(1)$ & $32(1)$ & $36(1)$ & $4(1)$ & $-1(1)$ & $1(1)$ \\
\hline$C(3)$ & $27(1)$ & $30(1)$ & $31(1)$ & $4(1)$ & $6(1)$ & $-4(1)$ \\
\hline$C(4)$ & $26(1)$ & $29(1)$ & $37(1)$ & $7(1)$ & $12(1)$ & $1(1)$ \\
\hline$C(5)$ & $49(2)$ & $51(2)$ & $51(2)$ & $15(2)$ & $25(2)$ & $5(2)$ \\
\hline$C(6)$ & $68(2)$ & $68(2)$ & $43(2)$ & $16(2)$ & $21(2)$ & $-7(2)$ \\
\hline$C(7)$ & $74(3)$ & $65(2)$ & $42(2)$ & $28(2)$ & $2(2)$ & $-7(2)$ \\
\hline$C(8)$ & $56(2)$ & $51(2)$ & $56(2)$ & $22(2)$ & $-1(2)$ & $9(2)$ \\
\hline $\mathrm{C}(1 \mathrm{~A})$ & $23(2)$ & $24(2)$ & $34(2)$ & $7(2)$ & $15(2)$ & $6(2)$ \\
\hline $\mathrm{C}(3 \mathrm{~A})$ & $22(2)$ & $24(2)$ & $36(2)$ & $4(2)$ & $10(2)$ & $6(2)$ \\
\hline $\mathrm{C}(2 \mathrm{~A})$ & $24(2)$ & $34(2)$ & $38(3)$ & $3(2)$ & $17(2)$ & $6(2)$ \\
\hline $\mathrm{N}(1 \mathrm{~A})$ & $33(1)$ & $26(2)$ & $47(2)$ & $0(1)$ & $12(1)$ & $1(1)$ \\
\hline $\mathrm{N}(2 \mathrm{~A})$ & $33(1)$ & $26(2)$ & $47(2)$ & $0(1)$ & $12(1)$ & $1(1)$ \\
\hline $\mathrm{C}(4 \mathrm{~A})$ & $50(4)$ & $40(3)$ & $60(4)$ & $-8(3)$ & $6(3)$ & $-18(3)$ \\
\hline$C(5 \mathrm{~A})$ & $33(2)$ & $47(5)$ & $103(4)$ & $48(4)$ & $12(2)$ & $1(3)$ \\
\hline$C(6 A)$ & $52(3)$ & $69(7)$ & 141(7) & $56(6)$ & $14(4)$ & $12(4)$ \\
\hline $\mathrm{C}(7 \mathrm{~A})$ & $97(7)$ & $79(6)$ & $85(6)$ & $-39(5)$ & $70(6)$ & $-18(5)$ \\
\hline $\mathrm{C}(8 \mathrm{~A})$ & $104(6)$ & $81(3)$ & 111(6) & $-36(4)$ & $27(5)$ & $43(4)$ \\
\hline $\mathrm{C}(9 \mathrm{~A})$ & $97(8)$ & $160(9)$ & $55(5)$ & $-8(5)$ & $31(5)$ & $21(7)$ \\
\hline$C(10 A)$ & $31(3)$ & $71(5)$ & $53(4)$ & $19(4)$ & $8(3)$ & $-14(3)$ \\
\hline$C(11 \mathrm{~A})$ & $33(2)$ & $47(5)$ & $103(4)$ & $48(4)$ & $12(2)$ & $1(3)$ \\
\hline $\mathrm{C}(12 \mathrm{~A})$ & $52(3)$ & $69(7)$ & $141(7)$ & $56(6)$ & $14(4)$ & $12(4)$ \\
\hline$C(13 \mathrm{~A})$ & $51(5)$ & $47(4)$ & $107(8)$ & $-38(4)$ & $9(5)$ & $-8(3)$ \\
\hline $\mathrm{C}(14 \mathrm{~A})$ & $97(8)$ & $160(9)$ & $55(5)$ & $-8(5)$ & $31(5)$ & 21(7) \\
\hline$C(15 A)$ & $104(6)$ & $81(3)$ & $111(6)$ & $-36(4)$ & $27(5)$ & $43(4)$ \\
\hline
\end{tabular}


Table 5. Hydrogen coordinates ( $\left.\times 10^{4}\right)$ and isotropic displacement parameters $\left(\AA^{2} \times 10^{3}\right)$ for rm08729b_0m_a.

\begin{tabular}{|c|c|c|c|c|}
\hline & $\mathrm{x}$ & $\mathrm{y}$ & $\mathrm{z}$ & $\mathrm{U}(\mathrm{eq})$ \\
\hline $\mathrm{H}(5)$ & $620(40)$ & $7160(60)$ & $2080(30)$ & $81(18)$ \\
\hline $\mathrm{H}(6)$ & $830(30)$ & $5550(50)$ & $3090(20)$ & $66(13)$ \\
\hline $\mathrm{H}(7)$ & $1800(30)$ & $3980(50)$ & $3290(30)$ & $69(13)$ \\
\hline $\mathrm{H}(8)$ & $2650(30)$ & $3770(50)$ & $2520(30)$ & $78(15)$ \\
\hline $\mathrm{H}(2 \mathrm{~A})$ & 923 & 10277 & -963 & 36 \\
\hline $\mathrm{H}(4 \mathrm{~A})$ & 1259 & 13090 & 1087 & 62 \\
\hline $\mathrm{H}(5 \mathrm{C})$ & 2002 & 11053 & 1004 & 94 \\
\hline $\mathrm{H}(5 \mathrm{~B})$ & 2372 & 12472 & 778 & 94 \\
\hline $\mathrm{H}(5 \mathrm{~A})$ & 1897 & 11408 & 151 & 94 \\
\hline $\mathrm{H}(6 \mathrm{C})$ & 809 & 13243 & -489 & 135 \\
\hline $\mathrm{H}(6 \mathrm{~B})$ & 1465 & 14265 & 11 & 135 \\
\hline $\mathrm{H}(6 \mathrm{~A})$ & 581 & 14340 & 66 & 135 \\
\hline $\mathrm{H}(7 \mathrm{~A})$ & -327 & 10814 & 926 & 93 \\
\hline $\mathrm{H}(8 \mathrm{C})$ & -452 & 13431 & 583 & 149 \\
\hline $\mathrm{H}(8 \mathrm{~B})$ & -43 & 13593 & 1451 & 149 \\
\hline $\mathrm{H}(8 \mathrm{~A})$ & -864 & 12780 & 1166 & 149 \\
\hline $\mathrm{H}(9 \mathrm{C})$ & 1093 & 10771 & 1753 & 153 \\
\hline $\mathrm{H}(9 \mathrm{~B})$ & 353 & 10586 & 2074 & 153 \\
\hline $\mathrm{H}(9 \mathrm{~A})$ & 765 & 12101 & 2102 & 153 \\
\hline $\mathrm{H}(10 \mathrm{~A})$ & -932 & 9011 & 314 & 63 \\
\hline $\mathrm{H}(11 \mathrm{C})$ & -1971 & 9730 & -748 & 94 \\
\hline $\mathrm{H}(11 \mathrm{~B})$ & -2300 & 8665 & -244 & 94 \\
\hline $\mathrm{H}(11 \mathrm{~A})$ & -2133 & 8107 & -988 & 94 \\
\hline $\mathrm{H}(12 \mathrm{C})$ & -1473 & 6312 & -153 & 135 \\
\hline $\mathrm{H}(12 \mathrm{~B})$ & -1059 & 6857 & 667 & 135 \\
\hline $\mathrm{H}(12 \mathrm{~A})$ & -538 & 6451 & 126 & 135 \\
\hline $\mathrm{H}(13 \mathrm{~A})$ & -997 & 6916 & -1243 & 85 \\
\hline $\mathrm{H}(14 \mathrm{C})$ & -1392 & 8957 & -1993 & 153 \\
\hline $\mathrm{H}(14 \mathrm{~B})$ & -1009 & 7761 & -2384 & 153 \\
\hline $\mathrm{H}(14 \mathrm{~A})$ & -508 & 9127 & -2035 & 153 \\
\hline $\mathrm{H}(15 \mathrm{C})$ & 591 & 7740 & -1068 & 149 \\
\hline
\end{tabular}


$\mathrm{H}(15 \mathrm{~B})$

182

6500

$-1620$

149

$\mathrm{H}(15 \mathrm{~A})$

295

6336

$-752$

149 
Table 6. Torsion angles $\left[^{\circ}\right]$ for $r m 08729 b \_0 m \_a$.

\begin{tabular}{|c|c|}
\hline $\mathrm{C}(4)-\mathrm{N}(1)-\mathrm{C}(1)-\mathrm{O}(1)$ & $-179.3(4)$ \\
\hline $\mathrm{Au}(1)-\mathrm{N}(1)-\mathrm{C}(1)-\mathrm{O}(1)$ & $0.0(5)$ \\
\hline $\mathrm{C}(4)-\mathrm{N}(1)-\mathrm{C}(1)-\mathrm{C}(2)$ & $-0.1(3)$ \\
\hline $\mathrm{Au}(1)-\mathrm{N}(1)-\mathrm{C}(1)-\mathrm{C}(2)$ & $179.19(18)$ \\
\hline $\mathrm{O}(1)-\mathrm{C}(1)-\mathrm{C}(2)-\mathrm{C}(3)$ & $179.2(4)$ \\
\hline $\mathrm{N}(1)-\mathrm{C}(1)-\mathrm{C}(2)-\mathrm{C}(3)$ & $0.0(3)$ \\
\hline $\mathrm{O}(1)-\mathrm{C}(1)-\mathrm{C}(2)-\mathrm{C}(8)$ & $-0.2(6)$ \\
\hline $\mathrm{N}(1)-\mathrm{C}(1)-\mathrm{C}(2)-\mathrm{C}(8)$ & $-179.4(3)$ \\
\hline $\mathrm{C}(8)-\mathrm{C}(2)-\mathrm{C}(3)-\mathrm{C}(5)$ & $1.3(5)$ \\
\hline$C(1)-C(2)-C(3)-C(5)$ & $-178.2(3)$ \\
\hline $\mathrm{C}(8)-\mathrm{C}(2)-\mathrm{C}(3)-\mathrm{C}(4)$ & $179.5(3)$ \\
\hline$C(1)-C(2)-C(3)-C(4)$ & $0.0(3)$ \\
\hline $\mathrm{C}(1)-\mathrm{N}(1)-\mathrm{C}(4)-\mathrm{O}(2)$ & 179.9(3) \\
\hline $\mathrm{Au}(1)-\mathrm{N}(1)-\mathrm{C}(4)-\mathrm{O}(2)$ & $0.6(5)$ \\
\hline $\mathrm{C}(1)-\mathrm{N}(1)-\mathrm{C}(4)-\mathrm{C}(3)$ & $0.1(3)$ \\
\hline $\mathrm{Au}(1)-\mathrm{N}(1)-\mathrm{C}(4)-\mathrm{C}(3)$ & $-179.20(17)$ \\
\hline $\mathrm{C}(2)-\mathrm{C}(3)-\mathrm{C}(4)-\mathrm{O}(2)$ & $-179.9(3)$ \\
\hline $\mathrm{C}(5)-\mathrm{C}(3)-\mathrm{C}(4)-\mathrm{O}(2)$ & $-1.9(6)$ \\
\hline $\mathrm{C}(2)-\mathrm{C}(3)-\mathrm{C}(4)-\mathrm{N}(1)$ & $-0.1(3)$ \\
\hline $\mathrm{C}(5)-\mathrm{C}(3)-\mathrm{C}(4)-\mathrm{N}(1)$ & $177.9(3)$ \\
\hline$C(2)-C(3)-C(5)-C(6)$ & $-0.3(5)$ \\
\hline$C(4)-C(3)-C(5)-C(6)$ & $-178.0(3)$ \\
\hline$C(3)-C(5)-C(6)-C(7)$ & $-0.5(6)$ \\
\hline$C(5)-C(6)-C(7)-C(8)$ & $0.3(7)$ \\
\hline $\mathrm{C}(6)-\mathrm{C}(7)-\mathrm{C}(8)-\mathrm{C}(2)$ & $0.6(6)$ \\
\hline$C(3)-C(2)-C(8)-C(7)$ & $-1.4(5)$ \\
\hline$C(1)-C(2)-C(8)-C(7)$ & $177.9(4)$ \\
\hline $\mathrm{N}(1 \mathrm{~A})-\mathrm{C}(1 \mathrm{~A})-\mathrm{C}(3 \mathrm{~A})-\mathrm{N}(2 \mathrm{~A})$ & $3.3(16)$ \\
\hline $\mathrm{C}(2 \mathrm{~A})-\mathrm{C}(1 \mathrm{~A})-\mathrm{C}(3 \mathrm{~A})-\mathrm{N}(2 \mathrm{~A})$ & $-177.5(10)$ \\
\hline$N(1 A)-C(1 A)-C(3 A)-C(2 A)$ & $-179.2(10)$ \\
\hline $\mathrm{N}(2 \mathrm{~A})-\mathrm{C}(3 \mathrm{~A})-\mathrm{C}(2 \mathrm{~A})-\mathrm{C}(1 \mathrm{~A})$ & $177.6(9)$ \\
\hline $\mathrm{N}(1 \mathrm{~A})-\mathrm{C}(1 \mathrm{~A})-\mathrm{C}(2 \mathrm{~A})-\mathrm{C}(3 \mathrm{~A})$ & 179.3(9) \\
\hline$C(2 A)-C(1 A)-N(1 A)-C(7 A)$ & $-175.6(7)$ \\
\hline $\mathrm{C}(3 \mathrm{~A})-\mathrm{C}(1 \mathrm{~A})-\mathrm{N}(1 \mathrm{~A})-\mathrm{C}(7 \mathrm{~A})$ & $3.1(11)$ \\
\hline $\mathrm{C}(2 \mathrm{~A})-\mathrm{C}(1 \mathrm{~A})-\mathrm{N}(1 \mathrm{~A})-\mathrm{C}(4 \mathrm{~A})$ & $-2.4(10)$ \\
\hline
\end{tabular}




$\begin{array}{lc}\mathrm{C}(3 \mathrm{~A})-\mathrm{C}(1 \mathrm{~A})-\mathrm{N}(1 \mathrm{~A})-\mathrm{C}(4 \mathrm{~A}) & 176.3(8) \\ \mathrm{C}(2 \mathrm{~A})-\mathrm{C}(3 \mathrm{~A})-\mathrm{N}(2 \mathrm{~A})-\mathrm{C}(10 \mathrm{~A}) & 176.1(7) \\ \mathrm{C}(1 \mathrm{~A})-\mathrm{C}(3 \mathrm{~A})-\mathrm{N}(2 \mathrm{~A})-\mathrm{C}(10 \mathrm{~A}) & -8.2(11) \\ \mathrm{C}(2 \mathrm{~A})-\mathrm{C}(3 \mathrm{~A})-\mathrm{N}(2 \mathrm{~A})-\mathrm{C}(13 \mathrm{~A}) & 3.8(10) \\ \mathrm{C}(1 \mathrm{~A})-\mathrm{C}(3 \mathrm{~A})-\mathrm{N}(2 \mathrm{~A})-\mathrm{C}(13 \mathrm{~A}) & 179.5(8) \\ \mathrm{C}(1 \mathrm{~A})-\mathrm{N}(1 \mathrm{~A})-\mathrm{C}(4 \mathrm{~A})-\mathrm{C}(5 \mathrm{~A}) & -52.9(3) \\ \mathrm{C}(7 \mathrm{~A})-\mathrm{N}(1 \mathrm{~A})-\mathrm{C}(4 \mathrm{~A})-\mathrm{C}(5 \mathrm{~A}) & 120.6 \\ \mathrm{C}(1 \mathrm{~A})-\mathrm{N}(1 \mathrm{~A})-\mathrm{C}(4 \mathrm{~A})-\mathrm{C}(6 \mathrm{~A}) & 72.4(3) \\ \mathrm{C}(7 \mathrm{~A})-\mathrm{N}(1 \mathrm{~A})-\mathrm{C}(4 \mathrm{~A})-\mathrm{C}(6 \mathrm{~A}) & -114.1 \\ \mathrm{C}(1 \mathrm{~A})-\mathrm{N}(1 \mathrm{~A})-\mathrm{C}(7 \mathrm{~A})-\mathrm{C}(9 \mathrm{~A}) & 114.9(3) \\ \mathrm{C}(4 \mathrm{~A})-\mathrm{N}(1 \mathrm{~A})-\mathrm{C}(7 \mathrm{~A})-\mathrm{C}(9 \mathrm{~A}) & -58.0 \\ \mathrm{C}(1 \mathrm{~A})-\mathrm{N}(1 \mathrm{~A})-\mathrm{C}(7 \mathrm{~A})-\mathrm{C}(8 \mathrm{~A}) & -119.9(3) \\ \mathrm{C}(4 \mathrm{~A})-\mathrm{N}(1 \mathrm{~A})-\mathrm{C}(7 \mathrm{~A})-\mathrm{C}(8 \mathrm{~A}) & 67.2 \\ \mathrm{C}(3 \mathrm{~A})-\mathrm{N}(2 \mathrm{~A})-\mathrm{C}(10 \mathrm{~A})-\mathrm{C}(12 \mathrm{~A}) & -119.9(3) \\ \mathrm{C}(13 \mathrm{~A})-\mathrm{N}(2 \mathrm{~A})-\mathrm{C}(10 \mathrm{~A})-\mathrm{C}(12 \mathrm{~A}) & 52.2 \\ \mathrm{C}(3 \mathrm{~A})-\mathrm{N}(2 \mathrm{~A})-\mathrm{C}(10 \mathrm{~A})-\mathrm{C}(11 \mathrm{~A}) & 116.4(3) \\ \mathrm{C}(13 \mathrm{~A})-\mathrm{N}(2 \mathrm{~A})-\mathrm{C}(10 \mathrm{~A})-\mathrm{C}(11 \mathrm{~A}) & -71.5 \\ \mathrm{C}(3 \mathrm{~A})-\mathrm{N}(2 \mathrm{~A})-\mathrm{C}(13 \mathrm{~A})-\mathrm{C}(14 \mathrm{~A}) & -72.1(3) \\ \mathrm{C}(10 \mathrm{~A})-\mathrm{N}(2 \mathrm{~A})-\mathrm{C}(13 \mathrm{~A})-\mathrm{C}(14 \mathrm{~A}) & 115.2 \\ \mathrm{C}(3 \mathrm{~A})-\mathrm{N}(2 \mathrm{~A})-\mathrm{C}(13 \mathrm{~A})-\mathrm{C}(15 \mathrm{~A}) & 52.9(3) \\ \mathrm{C}(10 \mathrm{~A})-\mathrm{N}(2 \mathrm{~A})-\mathrm{C}(13 \mathrm{~A})-\mathrm{C}(15 \mathrm{~A}) & -119.9\end{array}$

Symmetry transformations used to generate equivalent atoms:

$\# 1-x+1 / 2,-y+3 / 2,-z$ 


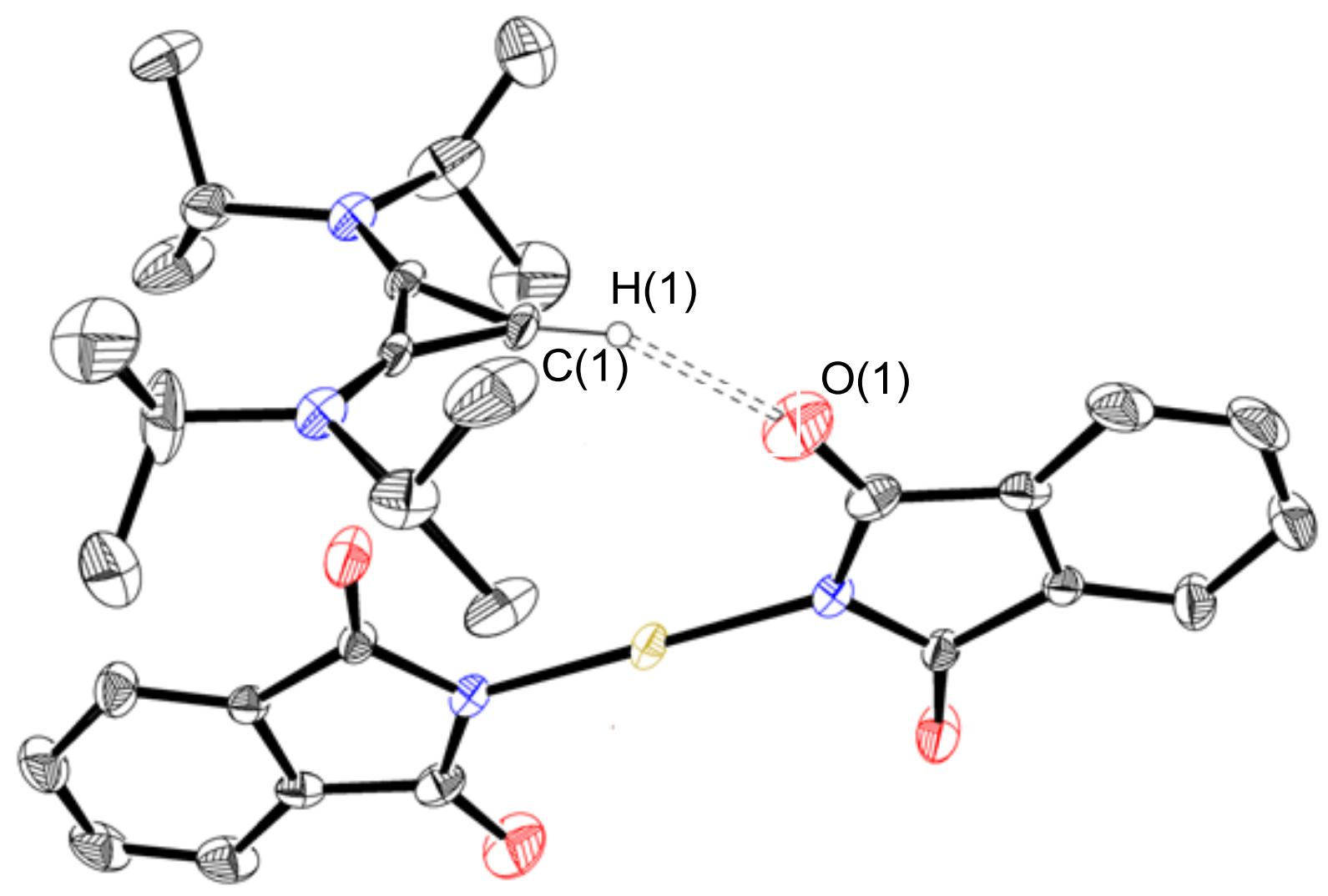

Figure 1. X-ray structure of $\mathbf{6}$ with $50 \%$ ellipsoid probability. 


\section{DFT calculated geometries and thermochemical data.}

$\underline{\text { TS2 }}$

\# opt=(calcfc,ts,noeigen) freq=noraman b3lyp/lanl2dz

Zero-point correction=

1.521591 (Hartree/Particle)

Thermal correction to Energy=

1.622800

Thermal correction to Enthalpy=

1.623744

Thermal correction to Gibbs Free Energy=

1.367591

Sum of electronic and zero-point Energies=

$-4208.801767$

Sum of electronic and thermal Energies=

$-4208.700558$

Sum of electronic and thermal Enthalpies=

$-4208.699614$

Sum of electronic and thermal Free Energies= $-4208.955768$

11

$\mathrm{N} \quad \begin{array}{llll}0.38652200 & 3.97614100 & -0.58512600\end{array}$

C $\quad 1.10294300 \quad 4.56409200 \quad-1.69192400$

C $\quad-0.60368900 \quad 4.81365900 \quad-0.24148600$

$\begin{array}{lllll}\mathrm{O} & -1.47109000 & 4.64628000 & 0.73430200\end{array}$

$\begin{array}{lllll}\text { O } & 2.08648000 & 4.04739600 & -2.24327800\end{array}$

$\mathrm{N}$

$\mathrm{H}$

$\mathrm{N}$

$\mathrm{N}$

$\mathrm{N}$

$\mathrm{N}$

C

C

C

C

C

$\mathrm{H}$

$\mathrm{H}$

C

$\mathrm{H}$

$\mathrm{H}$

C

$\mathrm{H}$

$\mathrm{H}$

$\mathrm{H}$ $\begin{array}{llll}-5.81526700 & -1.00664300 & -1.96762500\end{array}$

$\begin{array}{lll}0.62091400 & 2.71433700 & 0.01835700\end{array}$

$\begin{array}{llll}-3.04618400 & -3.24563900 & -2.62091200\end{array}$

$\begin{array}{lll}-5.27315100 & -4.05555100 & 0.27719300\end{array}$

$\begin{array}{lll}-7.35194200 & 0.76867800 & -1.93550800\end{array}$

$\begin{array}{lll}-6.17190600 & 0.47047600 & -0.04908400\end{array}$

$-5.13342600-2.05645200-1.51256300$

$-4.08270600 \quad-2.94475500-1.82891000$

$\begin{array}{llll}-4.93042600 & -3.22239700 & -0.72422500\end{array}$

$\begin{array}{lll}-6.39954300 & -0.00720900 & -1.31731200\end{array}$

$\begin{array}{lll}-7.61707500 & 2.00065100 & -1.16461700\end{array}$

$\begin{array}{lll}-8.69302500 & 2.19552100 & -1.09839300\end{array}$

$\begin{array}{llll}-7.12153300 & 2.85522000 & -1.64338200\end{array}$

$\begin{array}{lll}-6.98575300 & 1.68332400 & 0.21753700\end{array}$

$\begin{array}{llll}-6.33776300 & 2.49523400 & 0.55929400\end{array}$

$\begin{array}{lll}-7.74533200 & 1.47276800 & 0.98231700\end{array}$

$\begin{array}{llll}-7.65437100 & 0.69379200 & -3.36093900\end{array}$

$\begin{array}{lll}-7.10659300 & 1.46955400 & -3.91655900\end{array}$

$\begin{array}{lll}-7.34794200 & -0.28526200 & -3.73475800\end{array}$

$\begin{array}{lll}-8.73078400 & 0.82561300 & -3.52679700\end{array}$
C $\quad-5.07321300 \quad 0.11391700 \quad 0.85192000$

$\mathrm{H} \quad-4.35678100 \quad-0.54920100 \quad 0.36515600$

$\mathrm{H} \quad-4.54209900 \quad 1.02536700 \quad 1.13787900$

$\mathrm{H} \quad-5.44435500-0.37348000 \quad 1.76153400$

C $\quad-2.57463200 \quad-2.23104600 \quad-3.62711000$

$\mathrm{H} \quad-1.75533300 \quad-2.72194100 \quad-4.16589900$

C $\quad-2.01692600 \quad-0.97549300 \quad-2.92230400$

$\mathrm{H} \quad-1.56887000 \quad-0.30285700 \quad-3.66533800$

$\mathrm{H} \quad-2.80673200 \quad-0.41381800 \quad-2.41222300$

$\mathrm{H} \quad-1.25555100-1.24303200 \quad-2.18082000$

C $\quad-3.68438100 \quad-1.88129300 \quad-4.64350800$

$\mathrm{H} \quad-3.28669700 \quad-1.19362700 \quad-5.40079700$

$\mathrm{H} \quad-4.05455000 \quad-2.77934500 \quad-5.15432400$

$\mathrm{H} \quad-4.52502900-1.38371900 \quad-4.14718700$

C $\quad-2.43869300 \quad-4.61048400 \quad-2.58582600$

$\mathrm{H} \quad-2.89350000 \quad-5.11473700 \quad-1.72549400$

C $\quad-2.80111700 \quad-5.41428000 \quad-3.85605000$

$\mathrm{H} \quad-2.39681300 \quad-6.43239300 \quad-3.78636600$

$\mathrm{H} \quad-3.88821600 \quad-5.48262400 \quad-3.98142100$

$\mathrm{H} \quad-2.38128800-4.94894300 \quad-4.75668000$

C $\quad-0.91543100 \quad-4.56058400 \quad-2.34746300$

$\mathrm{H} \quad-0.53981400 \quad-5.57904500 \quad-2.18063600$

$\mathrm{H} \quad-0.38701900 \quad-4.14976500 \quad-3.21803300$

$\mathrm{H} \quad-0.68763200 \quad-3.94576100 \quad-1.47260100$

$\begin{array}{lllll}\text { C } & -6.67449200 & -4.08039700 & 0.80320900\end{array}$

$\mathrm{H} \quad-6.70161300 \quad-4.91928500 \quad 1.50774400$ 


\begin{tabular}{|c|c|c|c|c|c|c|c|}
\hline $\mathrm{C}$ & -7.05068500 & -2.79854100 & 1.57585300 & $\mathrm{H}$ & -1.55561400 & 3.70675300 & 1.16090600 \\
\hline $\mathrm{H}$ & -8.04464000 & -2.91637800 & 2.02641900 & $\mathrm{H}$ & -2.06461100 & 1.80956400 & 0.84329100 \\
\hline $\mathrm{H}$ & -6.33022700 & -2.59742700 & 2.37623800 & $\mathrm{C}$ & -2.49390300 & 2.00116100 & 2.88623900 \\
\hline $\mathrm{H}$ & -7.08589900 & -1.92897100 & 0.91015800 & $\mathrm{C}$ & -3.48997900 & 2.96738400 & 3.11496500 \\
\hline$\Omega$ & -7.68184600 & -4.37676300 & -0.33033400 & $\mathrm{C}$ & -2.38259400 & 0.86623600 & 3.71311000 \\
\hline $\mathrm{H}$ & -8.69633300 & -4.46183000 & 0.07877700 & $\mathrm{C}$ & -4.36174300 & 2.81327900 & 4.20898500 \\
\hline $\mathrm{H}$ & -7.68751400 & -3.57115100 & -1.07516700 & $\mathrm{H}$ & -3.59696800 & 3.81244300 & 2.44062900 \\
\hline $\mathrm{H}$ & -7.43609900 & -5.31614400 & -0.84007700 & $\mathrm{C}$ & -3.25291200 & 0.72665800 & 4.80842400 \\
\hline $\mathrm{C}$ & -4.23592400 & -4.96606600 & 0.86796700 & $\mathrm{H}$ & -1.64159500 & 0.10183000 & 3.49364400 \\
\hline $\mathrm{H}$ & -3.29185700 & -4.69687600 & 0.38305200 & $\mathrm{C}$ & -4.24009300 & 1.69964500 & 5.06270000 \\
\hline$C$ & -4.54654600 & -6.45098900 & 0.57858600 & $\mathrm{H}$ & -5.13055600 & 3.55972900 & 4.39181100 \\
\hline $\mathrm{H}$ & -3.73170800 & -7.08284400 & 0.95390000 & $\mathrm{H}$ & -3.16746000 & -0.14465500 & 5.45291900 \\
\hline $\mathrm{H}$ & -5.47049900 & -6.77714600 & 1.07324900 & $\mathrm{H}$ & -4.91047900 & 1.58740100 & 5.91115600 \\
\hline $\mathrm{H}$ & -4.65595300 & -6.63138500 & -0.49849500 & $\mathrm{~N}$ & -1.62037300 & 2.14296100 & 1.71983900 \\
\hline $\mathrm{C}$ & -4.04303600 & -4.68793400 & 2.37399500 & $\mathrm{C}$ & -0.61408500 & 6.03108100 & -1.08061100 \\
\hline $\mathrm{H}$ & -3.21890600 & -5.30284100 & 2.75435500 & $\mathrm{C}$ & -1.46061000 & 7.13678200 & -1.10215500 \\
\hline $\mathrm{H}$ & -3.77655000 & -3.63805600 & 2.53275900 & $\mathrm{C}$ & 0.44486200 & 5.87982800 & -1.99745200 \\
\hline $\mathrm{H}$ & -4.94234400 & -4.92694000 & 2.95654700 & $\mathrm{C}$ & -1.20158500 & 8.12466900 & -2.08318500 \\
\hline B & -0.85657000 & -2.43677700 & 1.12850400 & $\mathrm{H}$ & -2.29046200 & 7.22618600 & -0.40887700 \\
\hline $\mathrm{F}$ & -0.25750800 & -1.89510800 & 2.32605200 & $\mathrm{C}$ & 0.70470000 & 6.84240800 & -2.97043500 \\
\hline $\mathrm{F}$ & -0.94541600 & -3.88105200 & 1.25844100 & $\mathrm{C}$ & -0.13866700 & 7.98118600 & -2.99891400 \\
\hline $\mathrm{F}$ & 0.01925400 & -2.16597900 & 0.00085400 & $\mathrm{H}$ & -1.83736300 & 9.00395400 & -2.13997200 \\
\hline $\mathrm{F}$ & -2.15013400 & -1.88414100 & 0.91668900 & $\mathrm{H}$ & 1.51466500 & 6.72039900 & -3.68338200 \\
\hline B & -3.60459900 & 2.86876900 & -1.26698600 & $\mathrm{H}$ & 0.02876700 & 8.75433700 & -3.74403100 \\
\hline $\mathrm{F}$ & -2.72168000 & 3.81596600 & -1.86947800 & $\mathrm{Au}$ & 2.11894400 & 0.39455500 & 0.46283400 \\
\hline $\mathrm{F}$ & -4.25144400 & 3.44683100 & -0.10964400 & $\mathrm{~N}$ & 3.70531000 & -1.09021800 & 0.34584500 \\
\hline $\mathrm{F}$ & -2.78831500 & 1.74314300 & -0.76624700 & $\mathrm{C}$ & 4.95030500 & -0.95901200 & -0.16414000 \\
\hline $\mathrm{F}$ & -4.56926400 & 2.38268000 & -2.18656500 & $\mathrm{C}$ & 3.19358800 & -2.34861800 & 0.62531800 \\
\hline $\mathrm{C}$ & -0.23449600 & 1.88575700 & 1.76899000 & $\mathrm{C}$ & 5.91530700 & -0.13170300 & -0.78937400 \\
\hline $\mathrm{C}$ & 0.39624400 & 1.51091800 & 0.56481700 & $\mathrm{C}$ & 6.27059000 & -1.44236400 & -0.36181400 \\
\hline $\mathrm{H}$ & -0.32581200 & 1.13660800 & -0.17424100 & $\mathrm{~N}$ & 2.90925200 & -3.28964900 & -0.31181900 \\
\hline $\mathrm{C}$ & 0.47298200 & 2.32065900 & 3.00732800 & $\mathrm{~N}$ & 2.86800400 & -2.77489700 & 1.86109100 \\
\hline $\mathrm{C}$ & 1.50928600 & 1.54043900 & 3.56507600 & $\mathrm{~N}$ & 6.27382500 & 1.02058600 & -1.36683700 \\
\hline $\mathrm{C}$ & 0.12982300 & 3.54359800 & 3.63980400 & $\mathrm{~N}$ & 7.22358500 & -2.39174300 & -0.25224700 \\
\hline $\mathrm{C}$ & 2.18944900 & 1.96506400 & 4.71827300 & $\mathrm{C}$ & 2.19855800 & -4.44705300 & 0.30391800 \\
\hline $\mathrm{H}$ & 1.75313000 & 0.59200100 & 3.10234600 & $\mathrm{C}$ & 2.81594000 & -3.04985300 & -1.75508200 \\
\hline $\mathrm{C}$ & 0.82287600 & 3.97694200 & 4.78041300 & $\mathrm{C}$ & 2.21219100 & -4.10948800 & 1.82195900 \\
\hline $\mathrm{H}$ & -0.66135000 & 4.16876600 & 3.23884900 & $\mathrm{C}$ & 2.94996400 & -2.01506900 & 3.10806600 \\
\hline $\mathrm{C}$ & 1.85364600 & 3.18809400 & 5.32823200 & $\mathrm{C}$ & 5.24382100 & 2.06162900 & -1.72845600 \\
\hline $\mathrm{H}$ & 2.97314600 & 1.34320700 & 5.14442900 & $\mathrm{C}$ & 7.71758200 & 1.29728500 & -1.67609500 \\
\hline $\mathrm{H}$ & 0.55698800 & 4.92349300 & 5.24345100 & $\mathrm{C}$ & 7.09611500 & -3.53682700 & 0.70868500 \\
\hline $\mathrm{H}$ & 2.38002300 & 3.51966800 & 6.21979600 & $\mathrm{C}$ & 8.49811900 & -2.25718800 & -1.03939400 \\
\hline
\end{tabular}




\begin{tabular}{|c|c|c|c|c|c|c|c|}
\hline $\mathrm{H}$ & 2.71934200 & -5.38186000 & 0.07027000 & $\mathrm{H}$ & 4.19881900 & 2.12576400 & 0.19807600 \\
\hline $\mathrm{H}$ & 1.17498400 & -4.48799500 & -0.07589900 & $\mathrm{H}$ & 5.55735400 & 3.27144200 & 0.07285400 \\
\hline $\mathrm{H}$ & 3.27487100 & -2.09198700 & -2.00581300 & $\mathrm{H}$ & 3.43101000 & 2.25906500 & -2.88834800 \\
\hline $\mathrm{H}$ & 3.32684300 & -3.84829100 & -2.30595700 & $\mathrm{H}$ & 4.51376500 & 0.96498500 & -3.47514000 \\
\hline$\Pi$ & 1.76079900 & -3.01701800 & -2.05124500 & $\mathrm{H}$ & 3.51367900 & 0.73796500 & -2.01071600 \\
\hline 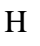 & 1.20124400 & -4.04659500 & 2.23103900 & $\mathrm{H}$ & 9.05020200 & 1.39788700 & -3.39841100 \\
\hline $\mathrm{H}$ & 2.80209800 & -4.82300800 & 2.40929000 & $\mathrm{H}$ & 7.67142600 & 0.29812700 & -3.63148500 \\
\hline $\mathrm{H}$ & 1.93701300 & -1.74973200 & 3.43118000 & $\mathrm{H}$ & 7.43912900 & 2.05461700 & -3.72065300 \\
\hline $\mathrm{H}$ & 3.43323100 & -2.62401800 & 3.88212600 & $\mathrm{H}$ & 9.27504000 & 2.73635400 & -1.22016200 \\
\hline $\mathrm{H}$ & 3.53474600 & -1.10735400 & 2.94517900 & $\mathrm{H}$ & 7.69578600 & 3.49141700 & -1.46645500 \\
\hline $\mathrm{H}$ & 5.78199400 & 2.78145100 & -2.35540700 & $\mathrm{H}$ & 8.03326200 & 2.61941900 & 0.04515000 \\
\hline $\mathrm{C}$ & 4.73248600 & 2.80464300 & -0.47798100 & $\mathrm{H}$ & 6.71171300 & -3.89841100 & 2.81653400 \\
\hline $\mathrm{C}$ & 4.11095500 & 1.45955900 & -2.58161100 & $\mathrm{H}$ & 7.37061100 & -2.28349300 & 2.48267300 \\
\hline $\mathrm{H}$ & 8.28561100 & 0.48980800 & -1.20341100 & $\mathrm{H}$ & 5.66820400 & -2.64672500 & 2.12486900 \\
\hline $\mathrm{C}$ & 7.98089400 & 1.25729800 & -3.19808700 & $\mathrm{H}$ & 6.15307700 & -5.49266500 & 0.87800000 \\
\hline $\mathrm{C}$ & 8.19980300 & 2.61798200 & -1.03801500 & $\mathrm{H}$ & 5.15075900 & -4.29132900 & 0.03445500 \\
\hline $\mathrm{H}$ & 8.10755400 & -3.94979300 & 0.79057700 & $\mathrm{H}$ & 6.53461000 & -5.03082800 & -0.79212400 \\
\hline $\mathrm{C}$ & 6.68412900 & -3.05543800 & 2.11542400 & $\mathrm{H}$ & 9.69409700 & -3.35484300 & -2.47760300 \\
\hline $\mathrm{C}$ & 6.17463800 & -4.65336800 & 0.17089900 & $\mathrm{H}$ & 9.04179000 & -4.38862200 & -1.20284900 \\
\hline $\mathrm{H}$ & 8.31142900 & -1.45892800 & -1.76528100 & $\mathrm{H}$ & 7.97693600 & -3.80375500 & -2.50421700 \\
\hline $\mathrm{C}$ & 8.81304300 & -3.53314400 & -1.84930000 & $\mathrm{H}$ & 10.57805100 & -1.67065400 & -0.74628700 \\
\hline $\mathrm{C}$ & 9.68153900 & -1.83982900 & -0.13726200 & $\mathrm{H}$ & 9.45980900 & -0.91676900 & 0.41232400 \\
\hline $\mathrm{H}$ & 4.02404200 & 3.57976000 & -0.78577900 & $\mathrm{H}$ & 9.92532300 & -2.61953300 & 0.59481600 \\
\hline
\end{tabular}

\# opt=calcfc freq=noraman b3lyp/lanl2dz

Zero-point correction=

Thermal correction to Energy=

Thermal correction to Enthalpy=
1.529528 (Hartree/Particle)

1.630586

1.631530

1.371168

$-4208.856130$

$-4208.755072$

$-4208.754128$

$-4209.014490$
11

$\begin{array}{lllll}\mathrm{N} & 0.24853800 & 4.15374600 & -1.43940200\end{array}$

$\begin{array}{lllll}\mathrm{C} & & 0.50385500 & 4.29499500 & -2.82745800\end{array}$

$\begin{array}{lllll}\text { C } & -0.46998200 & 5.23485400 & -0.91702500\end{array}$

$\begin{array}{lllll}\text { O } & -0.81176300 & 5.35831600 & 0.28423300\end{array}$
$\begin{array}{llll}\mathrm{O} & 1.13574400 & 3.47538200 & -3.51801600\end{array}$

$\mathrm{N} \quad \quad \quad-5.83867900 \quad-1.05475200 \quad-1.31912300$

$\mathrm{H} \quad 0.46478600 \quad 3.31000700 \quad-0.91532300$

$\mathrm{N} \quad \begin{array}{llll}-3.29572500 & -3.22216100 & -2.74492100\end{array}$

$\mathrm{N} \quad \begin{array}{llll}-5.15069500 & -4.40025200 & 0.31807700\end{array}$ 


\begin{tabular}{|c|c|c|c|c|c|c|c|}
\hline $\mathrm{N}$ & -7.15640000 & 0.78493500 & -0.69151600 & $\mathrm{C}$ & -6.36608600 & -3.18500300 & 2.17505400 \\
\hline $\mathrm{N}$ & -5.56876800 & 0.17902300 & 0.77929900 & $\mathrm{H}$ & -7.25463800 & -3.23803200 & 2.81775700 \\
\hline$C$ & -5.14240800 & -2.18567300 & -1.17099000 & $\mathrm{H}$ & -5.47602400 & -3.26321100 & 2.80900300 \\
\hline $\mathrm{C}$ & -4.19985800 & -3.04078800 & -1.77560800 & $\mathrm{H}$ & -6.36122700 & -2.20249700 & 1.69035700 \\
\hline $\mathrm{C}$ & -4.89483500 & -3.46200600 & -0.60806000 & $\mathrm{C}$ & -7.65332300 & -4.22640200 & 0.22655300 \\
\hline $\mathrm{C}$ & -6.14845300 & -0.11462400 & -0.43894500 & $\mathrm{H}$ & -8.56317700 & -4.25746300 & 0.83891000 \\
\hline $\mathrm{C}$ & -7.13183400 & 1.91048800 & 0.26202000 & $\mathrm{H}$ & -7.66382200 & -3.28804500 & -0.34100900 \\
\hline $\mathrm{H}$ & -8.13475000 & 2.10503600 & 0.65909500 & $\mathrm{H}$ & -7.68822900 & -5.06061800 & -0.48451100 \\
\hline $\mathrm{H}$ & -6.75658500 & 2.81619400 & -0.23041600 & $\mathrm{C}$ & -4.18199600 & -5.52181800 & 0.54957500 \\
\hline $\mathrm{C}$ & -6.14799000 & 1.41707700 & 1.35864300 & $\mathrm{H}$ & -3.28873300 & -5.27058300 & -0.03065200 \\
\hline $\mathrm{H}$ & -5.35942300 & 2.15425000 & 1.54216500 & $\mathrm{C}$ & -4.75111300 & -6.86846900 & 0.05169300 \\
\hline $\mathrm{H}$ & -6.65629200 & 1.19257200 & 2.30530000 & $\mathrm{H}$ & -4.00374800 & -7.66206800 & 0.17749100 \\
\hline $\mathrm{C}$ & -7.84748400 & 0.89031400 & -1.97103100 & $\mathrm{H}$ & -5.64547400 & -7.16340400 & 0.61589200 \\
\hline $\mathrm{H}$ & -7.43954100 & 1.72019600 & -2.56714200 & $\mathrm{H}$ & -5.02150400 & -6.81552600 & -1.01051100 \\
\hline $\mathrm{H}$ & -7.70421600 & -0.04037000 & -2.52411200 & $\mathrm{C}$ & -3.73369700 & -5.58436200 & 2.02600400 \\
\hline $\mathrm{H}$ & -8.92055600 & 1.05730300 & -1.81264800 & $\mathrm{H}$ & -2.96547300 & -6.35868100 & 2.13641000 \\
\hline $\mathrm{C}$ & -4.27891700 & -0.30729200 & 1.27737700 & $\mathrm{H}$ & -3.29315400 & -4.63001500 & 2.33291200 \\
\hline $\mathrm{H}$ & -3.95639300 & -1.20519000 & 0.75285600 & $\mathrm{H}$ & -4.56253500 & -5.83345100 & 2.70108600 \\
\hline $\mathrm{H}$ & -3.50256600 & 0.45710700 & 1.14282300 & $\mathrm{~B}$ & -0.82180600 & -3.30013800 & 0.70546400 \\
\hline $\mathrm{H}$ & -4.35864700 & -0.55035400 & 2.34440100 & $\mathrm{~F}$ & -0.42879200 & -3.05973800 & 2.07012800 \\
\hline $\mathrm{C}$ & -2.99531900 & -2.07587400 & -3.67130200 & $\mathrm{~F}$ & -0.85710200 & -4.73189500 & 0.46843900 \\
\hline $\mathrm{H}$ & -2.26342200 & -2.46881600 & -4.38604400 & $\mathrm{~F}$ & 0.17827100 & -2.73984200 & -0.18444800 \\
\hline $\mathrm{C}$ & -2.35053300 & -0.89978100 & -2.90500900 & $\mathrm{~F}$ & -2.10023700 & -2.73032500 & 0.44475100 \\
\hline $\mathrm{H}$ & -2.00642600 & -0.13746500 & -3.61566100 & $\mathrm{~B}$ & -3.46470200 & 3.05060300 & -0.82873500 \\
\hline $\mathrm{H}$ & -3.07094700 & -0.41764500 & -2.23449400 & $\mathrm{~F}$ & -2.80407400 & 3.90457600 & -1.75700200 \\
\hline $\mathrm{H}$ & -1.49670800 & -1.24007600 & -2.30809900 & $\mathrm{~F}$ & -3.96951800 & 3.79826400 & 0.29321800 \\
\hline $\mathrm{C}$ & -4.25102600 & -1.63346900 & -4.45514700 & $\mathrm{~F}$ & -2.43775900 & 2.12735200 & -0.25914400 \\
\hline $\mathrm{H}$ & -3.97917100 & -0.84888400 & -5.17272200 & $\mathrm{~F}$ & -4.48393400 & 2.28221000 & -1.43768100 \\
\hline $\mathrm{H}$ & -4.68860300 & -2.47226800 & -5.01105200 & $\mathrm{C}$ & 0.24875000 & 2.44249300 & 1.93136300 \\
\hline $\mathrm{H}$ & -5.00898800 & -1.22110600 & -3.77972200 & $\mathrm{C}$ & 0.36393300 & 1.28370000 & 1.23335300 \\
\hline $\mathrm{C}$ & -2.65963300 & -4.55735400 & -2.96483300 & $\mathrm{H}$ & -0.54651900 & 0.93823700 & 0.73263700 \\
\hline $\mathrm{H}$ & -2.92171900 & -5.15793900 & -2.08693200 & $\mathrm{C}$ & 1.25839200 & 3.15856700 & 2.76081800 \\
\hline $\mathrm{C}$ & -3.24511900 & -5.24922300 & -4.21792000 & $\mathrm{C}$ & 2.25692300 & 2.43152700 & 3.45339200 \\
\hline $\mathrm{H}$ & -2.81574100 & -6.25305700 & -4.33121000 & $\mathrm{C}$ & 1.24691600 & 4.56983600 & 2.89568300 \\
\hline $\mathrm{H}$ & -4.33478400 & -5.34628000 & -4.14174900 & $\mathrm{C}$ & 3.21762600 & 3.08780200 & 4.23883200 \\
\hline $\mathrm{H}$ & -3.01476500 & -4.68270200 & -5.12924000 & $\mathrm{H}$ & 2.26346600 & 1.34844900 & 3.38078600 \\
\hline $\mathrm{C}$ & -1.11926800 & -4.47075000 & -3.02389700 & $\mathrm{C}$ & 2.20845100 & 5.22629900 & 3.68344400 \\
\hline $\mathrm{H}$ & -0.70346600 & -5.48662700 & -3.03135400 & $\mathrm{H}$ & 0.50960000 & 5.17467700 & 2.37573600 \\
\hline $\mathrm{H}$ & -0.77785000 & -3.96622500 & -3.93713400 & $\mathrm{C}$ & 3.19985100 & 4.49125400 & 4.35931900 \\
\hline $\mathrm{H}$ & -0.72206000 & -3.94166300 & -2.15438700 & $\mathrm{H}$ & 3.96807100 & 2.50640800 & 4.76970600 \\
\hline $\mathrm{C}$ & -6.40626300 & -4.32405000 & 1.13242800 & $\mathrm{H}$ & 2.18106300 & 6.30972800 & 3.76895400 \\
\hline $\mathrm{H}$ & -6.45687900 & -5.27690000 & 1.67147400 & $\mathrm{H}$ & 3.93666300 & 5.00047700 & 4.97543600 \\
\hline
\end{tabular}




\begin{tabular}{|c|c|c|c|c|c|c|c|}
\hline $\mathrm{H}$ & -0.99553700 & 4.07413500 & 1.42881900 & $\mathrm{H}$ & 3.05853700 & -5.83794400 & 0.21685900 \\
\hline $\mathrm{H}$ & -1.68611600 & 2.60620300 & 1.17058700 & $\mathrm{H}$ & 1.43943700 & -5.13696400 & -0.08920500 \\
\hline $\mathrm{C}$ & -1.86273900 & 3.25245900 & 3.15255000 & $\mathrm{H}$ & 3.48997000 & -2.60088000 & -1.90164500 \\
\hline $\mathrm{C}$ & -2.71849000 & 4.35354200 & 3.30525200 & $\mathrm{H}$ & 3.38751600 & -4.35531900 & -2.14969200 \\
\hline $\mathrm{C}$ & -1.75149800 & 2.25967300 & 4.13595700 & $\mathrm{H}$ & 1.89444600 & -3.39060000 & -1.91347700 \\
\hline $\mathrm{C}$ & -3.46742400 & 4.47143600 & 4.49018100 & $\mathrm{H}$ & 1.19262500 & -4.71774900 & 2.21424800 \\
\hline $\mathrm{H}$ & -2.81518100 & 5.08970900 & 2.51210600 & $\mathrm{H}$ & 2.86745400 & -5.25398700 & 2.54170000 \\
\hline $\mathrm{C}$ & -2.50614400 & 2.38765000 & 5.31645600 & $\mathrm{H}$ & 1.50020400 & -2.25819100 & 3.41901600 \\
\hline $\mathrm{H}$ & -1.09020200 & 1.41019400 & 3.99031500 & $\mathrm{H}$ & 2.96984800 & -3.06538500 & 4.04960800 \\
\hline $\mathrm{C}$ & -3.36064400 & 3.49251500 & 5.49738100 & $\mathrm{H}$ & 3.10798500 & -1.52897500 & 3.15129600 \\
\hline $\mathrm{H}$ & -4.13292700 & 5.32011400 & 4.62174000 & $\mathrm{H}$ & 4.73374700 & 2.25237200 & -2.90468600 \\
\hline $\mathrm{H}$ & -2.42495400 & 1.62882100 & 6.09007400 & $\mathrm{C}$ & 3.85346000 & 2.57811100 & -0.96716900 \\
\hline $\mathrm{H}$ & -3.93836500 & 3.58916300 & 6.41282800 & $\mathrm{C}$ & 3.21215100 & 0.76534200 & -2.66358500 \\
\hline $\mathrm{N}$ & -1.10616300 & 3.12743900 & 1.87022200 & $\mathrm{H}$ & 7.59788000 & 0.60010700 & -1.53079500 \\
\hline $\mathrm{C}$ & -0.68382400 & 6.17358900 & -2.05064600 & $\mathrm{C}$ & 7.08273800 & 0.90126900 & -3.60744200 \\
\hline $\mathrm{C}$ & -1.34303800 & 7.40132600 & -2.09067000 & $\mathrm{C}$ & 7.21683500 & 2.68564200 & -1.77550800 \\
\hline $\mathrm{C}$ & -0.10330000 & 5.60667200 & -3.20502700 & $\mathrm{H}$ & 8.19090500 & -3.49191200 & 1.02940600 \\
\hline $\mathrm{C}$ & -1.40778900 & 8.06421300 & -3.33824200 & $\mathrm{C}$ & 6.62636100 & -2.70117000 & 2.26002800 \\
\hline $\mathrm{H}$ & -1.79929000 & 7.82179200 & -1.19994800 & $\mathrm{C}$ & 6.40886700 & -4.59266300 & 0.53993700 \\
\hline $\mathrm{C}$ & -0.16245700 & 6.25110800 & -4.44072300 & $\mathrm{H}$ & 7.94658500 & -1.37668800 & -1.84910200 \\
\hline $\mathrm{C}$ & -0.82720700 & 7.49869800 & -4.49377600 & $\mathrm{C}$ & 8.77308600 & -3.33878300 & -1.67940300 \\
\hline $\mathrm{H}$ & -1.91808300 & 9.02058400 & -3.41291000 & $\mathrm{C}$ & 9.38789400 & -1.31396300 & -0.23907900 \\
\hline $\mathrm{H}$ & 0.27829600 & 5.80565000 & -5.32753800 & $\mathrm{H}$ & 3.06756700 & 3.22961700 & -1.36586900 \\
\hline $\mathrm{H}$ & -0.89896500 & 8.02950200 & -5.43926400 & $\mathrm{H}$ & 3.43150400 & 2.01068000 & -0.12948100 \\
\hline $\mathrm{Au}$ & 1.90761700 & 0.02870300 & 0.93010000 & $\mathrm{H}$ & 4.66251300 & 3.21102500 & -0.58711200 \\
\hline $\mathrm{N}$ & 3.43501000 & -1.44492000 & 0.53368900 & $\mathrm{H}$ & 2.44774900 & 1.41934900 & -3.09786100 \\
\hline $\mathrm{C}$ & 4.60173000 & -1.17707100 & -0.10494000 & $\mathrm{H}$ & 3.58852100 & 0.10141500 & -3.45298800 \\
\hline $\mathrm{C}$ & 3.06951900 & -2.75468500 & 0.77390700 & $\mathrm{H}$ & 2.72689300 & 0.16113400 & -1.88799300 \\
\hline $\mathrm{C}$ & 5.37481900 & -0.29580700 & -0.89851100 & $\mathrm{H}$ & 8.10847200 & 1.13753600 & -3.91656800 \\
\hline $\mathrm{C}$ & 5.96443600 & -1.47674800 & -0.34816000 & $\mathrm{H}$ & 6.89283100 & -0.15768100 & -3.82293000 \\
\hline $\mathrm{N}$ & 3.03454400 & -3.73727200 & -0.17261700 & $\mathrm{H}$ & 6.40494000 & 1.49876900 & -4.22856400 \\
\hline $\mathrm{N}$ & 2.67998300 & -3.22021600 & 1.97554100 & $\mathrm{H}$ & 8.25562200 & 2.89764500 & -2.05658500 \\
\hline $\mathrm{N}$ & 5.52408100 & 0.80981100 & -1.63652700 & $\mathrm{H}$ & 6.57861800 & 3.38286100 & -2.32948300 \\
\hline $\mathrm{N}$ & 7.05021200 & -2.25377600 & -0.18364500 & $\mathrm{H}$ & 7.10508900 & 2.88759200 & -0.70437600 \\
\hline $\mathrm{C}$ & 2.41004000 & -4.97229800 & 0.38730000 & $\mathrm{H}$ & 6.81282800 & -3.42776200 & 3.05994800 \\
\hline $\mathrm{C}$ & 2.94590400 & -3.50393900 & -1.62062100 & $\mathrm{H}$ & 7.15504800 & -1.77407100 & 2.51175200 \\
\hline $\mathrm{C}$ & 2.23319000 & -4.63633400 & 1.89428400 & $\mathrm{H}$ & 5.55143000 & -2.49199100 & 2.24411900 \\
\hline $\mathrm{C}$ & 2.55794600 & -2.46937500 & 3.22589600 & $\mathrm{H}$ & 6.52364700 & -5.32282200 & 1.35137400 \\
\hline $\mathrm{C}$ & 4.34401300 & 1.63707900 & -2.08603800 & $\mathrm{H}$ & 5.34014600 & -4.42772000 & 0.37077800 \\
\hline $\mathrm{C}$ & 6.89611300 & 1.21259000 & -2.10531100 & $\mathrm{H}$ & 6.83531300 & -5.02803000 & -0.36989600 \\
\hline $\mathrm{C}$ & 7.12250700 & -3.27619800 & 0.91685700 & $\mathrm{H}$ & 9.60943800 & -3.11179500 & -2.35148500 \\
\hline $\mathrm{C}$ & 8.27176500 & -2.03006600 & -1.03305100 & $\mathrm{H}$ & 9.13827600 & -4.05531300 & -0.93449100 \\
\hline
\end{tabular}




$\begin{array}{llllllll}\mathrm{H} & 7.98353300 & -3.81914800 & -2.26836400 & \mathrm{H} & 9.03048500 & -0.37034800 & 0.19120200 \\ \mathrm{H} & 10.23590300 & -1.09232900 & -0.89850500 & \mathrm{H} & 9.76432300 & -1.93846200 & 0.58036600\end{array}$

\# opt=calcfc freq=noraman b3lyp/lanl2dz

Zero-point correction $=$

Thermal correction to Energy=

Thermal correction to Enthalpy=
1.527897 (Hartree/Particle)

\subsection{2}

1.630626

Thermal correction to Gibbs Free Energy= $\quad 1.369100$

Sum of electronic and zero-point Energies= $-4208.852370$

Sum of electronic and thermal Energies= $-4208.750585$

Sum of electronic and thermal Enthalpies= $-4208.749641$

Sum of electronic and thermal Free Energies= $-4209.011167$

11

$\mathrm{N}$

C

C

$\mathrm{O}$

$\mathrm{O}$

N

H

$\mathrm{N}$

$\mathrm{N}$

$\mathrm{N}$

$\mathrm{N}$

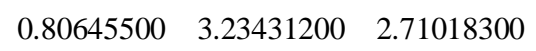

C $\quad 3.67341300 \quad-1.05170900 \quad-2.30703700$

$\mathrm{H} \quad 3.57240400 \quad-1.68174900 \quad-1.42439000$

$\mathrm{H} \quad 2.86784200-0.30756200 \quad-2.29071500$

$\mathrm{H} \quad 3.54943100 \quad-1.69264700 \quad-3.18899000$

C $\quad 3.39568200 \quad-0.71237900 \quad 2.88750600$

$\mathrm{H} \quad 2.78026300 \quad-0.78536400 \quad 3.79167000$

$\begin{array}{llll}\text { C } & 2.53990200 & -0.07963500 & 1.76730400\end{array}$

$\mathrm{H} \quad 2.11990300 \quad 0.87389500 \quad 2.11055900$

$\mathrm{H} \quad 3.14661400 \quad 0.12857900 \quad 0.87863400$

$\mathrm{H} \quad \begin{array}{llll}1.71382700 & -0.74060400 & 1.48276900\end{array}$

C $\quad 4.64608800 \quad 0.13402000 \quad 3.21078700$

$\mathrm{H} \quad 4.34209300 \quad 1.14644800 \quad 3.50581200$

$\mathrm{H} \quad 5.22301500 \quad-0.30765700 \quad 4.03309100$

$\mathrm{H} \quad \begin{array}{llll}\mathrm{H} & 5.29385300 & 0.22406800 & 2.33126500\end{array}$

C $\quad 3.26975500 \quad-3.25400400 \quad 3.40244100$

$\mathrm{H} \quad 3.66304300 \quad-4.16861500 \quad 2.94387200$

C $\quad 3.84491600 \quad-3.16730200 \quad 4.83439900$

$\mathrm{H} \quad 3.52460000 \quad-4.03945800 \quad 5.41850700$

$\mathrm{H} \quad 4.94105600 \quad-3.14553000 \quad 4.81744900$

$\mathrm{H} \quad 3.49135700 \quad-2.27024300 \quad 5.35769000$

C $\quad 1.72803600 \quad-3.34156200 \quad 3.39652700$

$\mathrm{H} \quad 1.40812100 \quad-4.23961000 \quad 3.94156900$

$\mathrm{H} \quad 1.27539000 \quad-2.47370600 \quad 3.89289500$

$\mathrm{H} \quad 1.34183300 \quad-3.38981600 \quad 2.37469200$

C $\quad 6.28479400 \quad-4.69195200 \quad-1.07269800$

$\mathrm{H} \quad 6.34506400 \quad-5.77968400 \quad-1.19130000$ 


\begin{tabular}{|c|c|c|c|c|c|c|c|}
\hline $\mathrm{C}$ & 5.85354300 & -4.08464400 & -2.42652900 & $\mathrm{H}$ & 2.48848400 & 4.23371500 & 1.02629500 \\
\hline $\mathrm{H}$ & 6.57168500 & -4.36943100 & -3.20647700 & $\mathrm{H}$ & 0.77408000 & 2.61628900 & -2.39539100 \\
\hline$H$ & 4.86132400 & -4.44369400 & -2.72114400 & $\mathrm{C}$ & -0.15248900 & 3.87909700 & -3.77888700 \\
\hline $\mathrm{H}$ & 5.82550900 & -2.99026000 & -2.38089600 & $\mathrm{C}$ & 1.05130500 & 4.36268500 & -4.33334000 \\
\hline $\mathrm{C}$ & 7.67515400 & -4.18180600 & -0.63454200 & $\mathrm{C}$ & -1.36879700 & 4.00966700 & -4.48084400 \\
\hline $\mathrm{H}$ & 8.42020800 & -4.41992700 & -1.40396600 & $\mathrm{C}$ & 1.02577000 & 4.99973100 & -5.58647100 \\
\hline $\mathrm{H}$ & 7.67382600 & -3.09417300 & -0.49417300 & $\mathrm{H}$ & 1.98777900 & 4.25640200 & -3.79171300 \\
\hline $\mathrm{H}$ & 7.98852900 & -4.65164300 & 0.30548400 & $\mathrm{C}$ & -1.38104600 & 4.65327700 & -5.72957000 \\
\hline C & 4.44118900 & -5.61716300 & 0.49687700 & $\mathrm{H}$ & -2.28918100 & 3.61471100 & -4.06207900 \\
\hline$H$ & 3.70847700 & -5.17595800 & 1.17976500 & $\mathrm{C}$ & -0.18675200 & 5.15260500 & -6.28625900 \\
\hline $\mathrm{C}$ & 5.30432900 & -6.63173000 & 1.27942700 & $\mathrm{H}$ & 1.95196200 & 5.37894600 & -6.00975100 \\
\hline 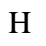 & 4.66734200 & -7.41905400 & 1.70218200 & $\mathrm{H}$ & -2.31879700 & 4.75871900 & -6.26906200 \\
\hline $\mathrm{H}$ & 6.04389700 & -7.11976200 & 0.63187500 & $\mathrm{H}$ & -0.20101800 & 5.64891700 & -7.25292400 \\
\hline $\mathrm{H}$ & 5.84095800 & -6.14240800 & 2.10167000 & $\mathrm{~N}$ & -0.07479700 & 3.18767000 & -2.51859600 \\
\hline $\mathrm{C}$ & 3.63934000 & -6.28060000 & -0.64356300 & $\mathrm{C}$ & 1.96506800 & 4.52544900 & 4.32006200 \\
\hline 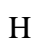 & 3.00892300 & -7.07790800 & -0.23010800 & $\mathrm{C}$ & 2.85178200 & 5.39271600 & 4.95507700 \\
\hline $\mathrm{H}$ & 2.98402000 & -5.54892000 & -1.12528000 & $\mathrm{C}$ & 0.96292500 & 3.83181200 & 5.02831700 \\
\hline $\mathrm{H}$ & 4.29560400 & -6.73495900 & -1.39699800 & $\mathrm{C}$ & 2.70543300 & 5.55365500 & 6.35629700 \\
\hline B & 0.75839400 & -3.19273800 & -0.59412800 & $\mathrm{H}$ & 3.62102500 & 5.92174900 & 4.40120100 \\
\hline $\mathrm{F}$ & 0.56663100 & -2.55520600 & -1.87663900 & $\mathrm{C}$ & 0.81086100 & 3.98205800 & 6.40480400 \\
\hline $\mathrm{F}$ & 0.34306300 & -4.57931300 & -0.70322300 & $\mathrm{C}$ & 1.70450000 & 4.86157200 & 7.06799500 \\
\hline $\mathrm{F}$ & -0.06561500 & -2.53290700 & 0.39160100 & $\mathrm{H}$ & 3.37522400 & 6.22115100 & 6.89209200 \\
\hline $\mathrm{F}$ & 2.13038200 & -3.14378400 & -0.20831000 & $\mathrm{H}$ & 0.03813100 & 3.44672300 & 6.94893500 \\
\hline $\mathrm{B}$ & 3.25588900 & 2.96588600 & -1.35879100 & $\mathrm{H}$ & 1.61829300 & 5.00640100 & 8.14178800 \\
\hline $\mathrm{F}$ & 4.24050900 & 2.33604600 & -0.57368600 & $\mathrm{Au}$ & -1.99249000 & 0.53910300 & -0.71138400 \\
\hline $\mathrm{F}$ & 2.43893900 & 3.85116800 & -0.52358700 & $\mathrm{~N}$ & -3.19513400 & -1.23739900 & -0.88601400 \\
\hline $\mathrm{F}$ & 2.33085400 & 1.97486900 & -1.92187300 & $\mathrm{C}$ & -4.22268000 & -1.46869800 & -0.01848200 \\
\hline $\mathrm{F}$ & 3.80743900 & 3.71241600 & -2.43750400 & $\mathrm{C}$ & -2.78408300 & -2.23683600 & -1.73352700 \\
\hline $\mathrm{C}$ & -0.92977900 & 3.17659800 & -1.48471300 & $\mathrm{C}$ & -4.83509100 & -1.21125900 & 1.23043600 \\
\hline 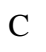 & -0.72364800 & 2.20037100 & -0.42076000 & $\mathrm{C}$ & -5.50120400 & -2.00624200 & 0.23811900 \\
\hline $\mathrm{H}$ & 0.27916600 & 1.76209300 & -0.43385500 & $\mathrm{~N}$ & -2.67036400 & -3.55033400 & -1.39600500 \\
\hline $\mathrm{C}$ & -1.99074000 & 4.21918700 & -1.36717900 & $\mathrm{~N}$ & -2.41733100 & -2.03549300 & -3.02287200 \\
\hline $\mathrm{C}$ & -3.31469800 & 3.89603300 & -0.99764700 & $\mathrm{~N}$ & -4.85005500 & -0.66571000 & 2.44763400 \\
\hline $\mathrm{C}$ & -1.63044000 & 5.57907700 & -1.53281900 & $\mathrm{~N}$ & -6.57929100 & -2.71246300 & -0.14468800 \\
\hline $\mathrm{C}$ & -4.26869800 & 4.91199900 & -0.81709000 & $\mathrm{C}$ & -2.14286600 & -4.34761200 & -2.54239100 \\
\hline $\mathrm{H}$ & -3.59363400 & 2.85343200 & -0.87259600 & $\mathrm{C}$ & -2.69252500 & -4.09963100 & -0.03184500 \\
\hline $\mathrm{C}$ & -2.58129700 & 6.59197700 & -1.33330500 & $\mathrm{C}$ & -1.80907800 & -3.26258000 & -3.60237200 \\
\hline $\mathrm{H}$ & -0.60876100 & 5.84126500 & -1.79123700 & $\mathrm{C}$ & -2.40684200 & -0.77600600 & -3.76372300 \\
\hline $\mathrm{C}$ & -3.90542400 & 6.26255100 & -0.98196400 & $\mathrm{C}$ & -3.64462100 & 0.05236500 & 3.01847300 \\
\hline $\mathrm{H}$ & -5.28920700 & 4.65309600 & -0.54634300 & $\mathrm{C}$ & -6.08170800 & -0.78313400 & 3.30551100 \\
\hline & -2.29078400 & 7.63251900 & -1.44961200 & $\mathrm{C}$ & -6.84735300 & -2.99597000 & -1.59865400 \\
\hline & -4.64204500 & 7.04852700 & -0.83604200 & $\mathrm{C}$ & -7.61968300 & -3.10919200 & 0.86727400 \\
\hline
\end{tabular}




\begin{tabular}{|c|c|c|c|c|c|c|c|}
\hline $\mathrm{H}$ & -2.90100800 & -5.05958000 & -2.89119200 & $\mathrm{H}$ & -3.38487100 & 1.47453000 & 1.38160400 \\
\hline $\mathrm{H}$ & -1.24884000 & -4.88357700 & -2.21832900 & $\mathrm{H}$ & -4.43863600 & 2.07180800 & 2.68494100 \\
\hline 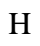 & -2.39450700 & -3.32996200 & 0.68314000 & $\mathrm{H}$ & -1.53647500 & -0.22275800 & 3.34969400 \\
\hline $\mathrm{H}$ & -3.68140200 & -4.50182200 & 0.22509800 & $\mathrm{H}$ & -2.43358900 & -1.75979500 & 3.25896800 \\
\hline $\mathrm{H}$ & -1.94238200 & -4.89150700 & 0.02751000 & $\mathrm{H}$ & -2.04789300 & -0.83979500 & 1.78230600 \\
\hline $\mathrm{H}$ & -0.72950500 & -3.12300700 & -3.70708400 & $\mathrm{H}$ & -6.78997200 & -1.93693900 & 5.01407000 \\
\hline 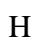 & -2.25808300 & -3.47392900 & -4.57856100 & $\mathrm{H}$ & -5.57650000 & -2.79274200 & 4.03423900 \\
\hline $\mathrm{H}$ & -1.40435800 & -0.32872400 & -3.76325000 & $\mathrm{H}$ & -5.08219600 & -1.48869700 & 5.13077800 \\
\hline $\mathrm{H}$ & -2.70927700 & -0.96851400 & -4.80020700 & $\mathrm{H}$ & -7.49184800 & 0.44117100 & 4.39924900 \\
\hline $\mathrm{H}$ & -3.10786100 & -0.06773500 & -3.31623100 & $\mathrm{H}$ & -5.82965300 & 1.02510500 & 4.54111100 \\
\hline $\mathrm{H}$ & -3.84282800 & 0.11210900 & 4.09445900 & $\mathrm{H}$ & -6.73691600 & 1.29355700 & 3.03638000 \\
\hline $\mathrm{C}$ & -3.53772600 & 1.48744000 & 2.46710600 & $\mathrm{H}$ & -6.93617100 & -1.95514200 & -3.50062800 \\
\hline $\mathrm{C}$ & -2.34454100 & -0.75197100 & 2.83306300 & $\mathrm{H}$ & -7.18260700 & -0.88862700 & -2.10296200 \\
\hline $\mathrm{H}$ & -6.87141400 & -1.15094700 & 2.64338400 & $\mathrm{H}$ & -5.55165900 & -1.46792400 & -2.51138200 \\
\hline $\mathrm{C}$ & -5.86575100 & -1.81382500 & 4.43620000 & $\mathrm{H}$ & -6.34500100 & -4.43018100 & -3.15414100 \\
\hline $\mathrm{C}$ & -6.55345700 & 0.58176500 & 3.84891500 & $\mathrm{H}$ & -4.98753400 & -4.04636300 & -2.07508100 \\
\hline $\mathrm{H}$ & -7.91659500 & -3.23129400 & -1.64511800 & $\mathrm{H}$ & -6.29024100 & -5.11612000 & -1.51915700 \\
\hline $\mathrm{C}$ & -6.61059900 & -1.74731100 & -2.47439100 & $\mathrm{H}$ & -8.67529700 & -4.86354300 & 1.57563300 \\
\hline $\mathrm{C}$ & -6.06641300 & -4.22392200 & -2.11292900 & $\mathrm{H}$ & -8.48446600 & -4.84715200 & -0.17999300 \\
\hline H & -7.14706300 & -2.96464300 & 1.84443000 & $\mathrm{H}$ & -7.09484000 & -5.23865000 & 0.86132200 \\
\hline C & -7.98383500 & -4.60505500 & 0.76465400 & $\mathrm{H}$ & -9.57349500 & -2.45603700 & 1.58079600 \\
\hline $\mathrm{C}$ & -8.86807500 & -2.20254000 & 0.78006400 & $\mathrm{H}$ & -8.60104600 & -1.14323600 & 0.87964800 \\
\hline $\mathrm{H}$ & -2.67430600 & 1.98088200 & 2.92484300 & $\mathrm{H}$ & -9.39466100 & -2.33154300 & -0.17346800 \\
\hline
\end{tabular}

\section{Optimized structure of 6 (all of the atoms except for the hydrogens frozen)}

\# opt=calcfc freq=noraman b3lyp/lanl2dz

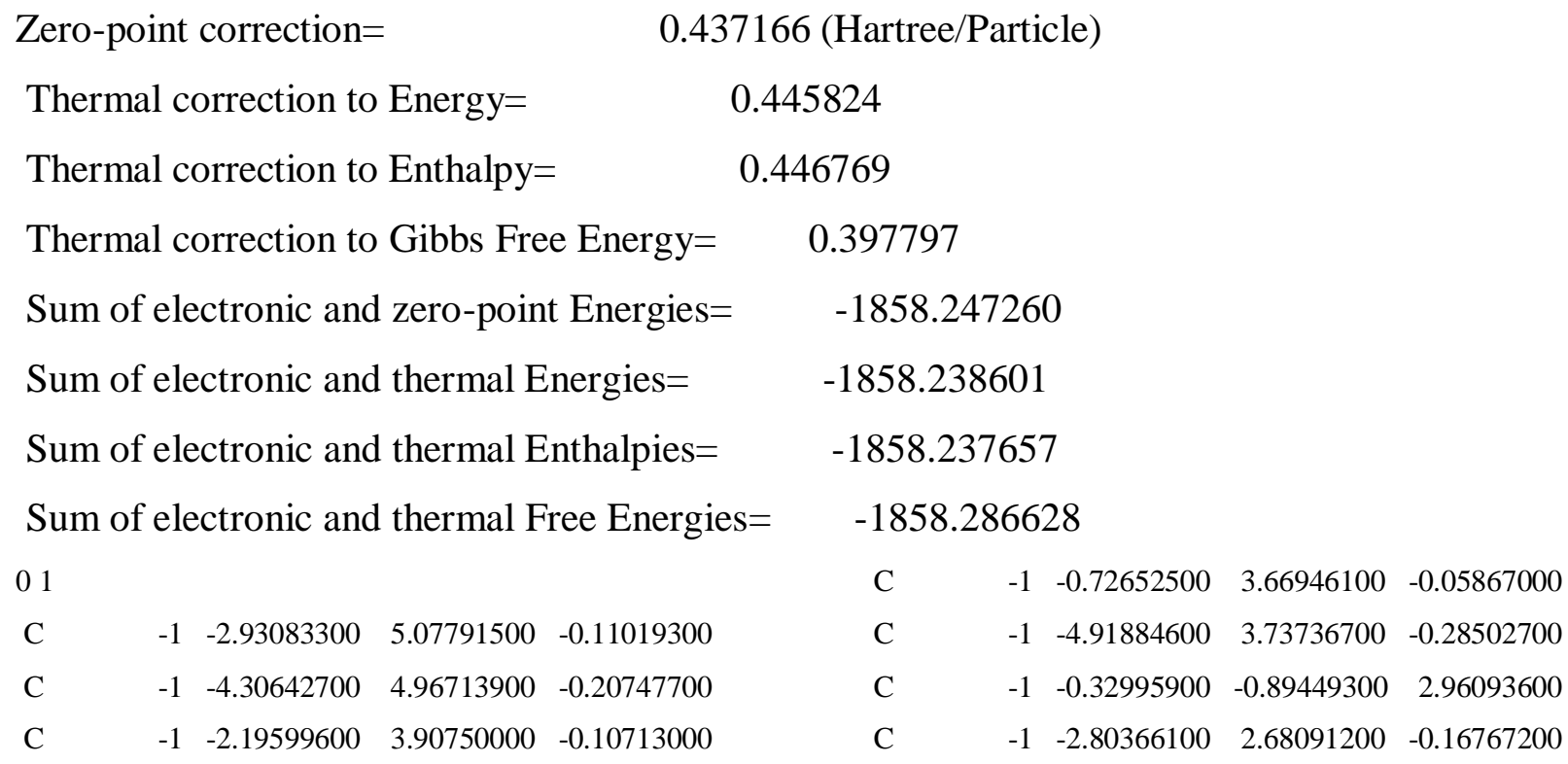




\begin{tabular}{|c|c|c|c|c|c|c|c|c|c|}
\hline $\mathrm{C}$ & -1 & 6.68744200 & 0.18296800 & 0.10652000 & $\mathrm{H}$ & 0 & -4.91699300 & 5.86740900 & -0.22382400 \\
\hline $\mathrm{C}$ & -1 & 4.23945800 & 1.09518300 & 0.00360100 & $\mathrm{H}$ & 0 & -6.00258400 & 3.68345000 & -0.3643290 \\
\hline $\mathrm{C}$ & -1 & -4.17220700 & 2.56194900 & -0.26104200 & $\mathrm{H}$ & 0 & -0.83138700 & 0.01242700 & 2.60879200 \\
\hline $\mathrm{C}$ & -1 & -4.04551300 & -0.74533500 & 2.77936300 & $\mathrm{H}$ & 0 & 0.17924300 & -0.65526100 & 3.9039280 \\
\hline $\mathrm{C}$ & -1 & 5.31889600 & 0.06400500 & 0.01315000 & $\mathrm{H}$ & 0 & 0.44542900 & -1.14454500 & 2.2323700 \\
\hline $\mathrm{C}$ & -1 & 7.43397800 & -0.99313000 & 0.12978000 & $\mathrm{H}$ & 0 & 7.15816500 & 1.16039900 & 0.16464600 \\
\hline $\mathrm{C}$ & -1 & -1.31689200 & -2.03588400 & 3.18454600 & $\mathrm{H}$ & 0 & -4.65033200 & 1.58719000 & -0.3217340 \\
\hline $\mathrm{C}$ & -1 & -1.72430700 & 1.64901300 & -0.15743500 & $\mathrm{H}$ & 0 & -5.13667300 & -0.62861200 & 2.7534070 \\
\hline $\mathrm{C}$ & -1 & -0.62007300 & -3.31881000 & 3.55062900 & $\mathrm{H}$ & 0 & -3.75041800 & -0.71602800 & 3.8345710 \\
\hline $\mathrm{C}$ & -1 & -3.65949700 & -2.02971900 & 2.10109700 & $\mathrm{H}$ & 0 & -3.60515600 & 0.12188700 & 2.27450200 \\
\hline $\mathrm{C}$ & -1 & 4.71123100 & -1.16258200 & -0.04738600 & $\mathrm{H}$ & 0 & 8.51752700 & -0.93802400 & 0.20751000 \\
\hline $\mathrm{C}$ & -1 & 6.82166300 & -2.22222100 & 0.05295500 & $\mathrm{H}$ & 0 & -1.99220600 & -1.75776800 & 3.9992220 \\
\hline $\mathrm{C}$ & -1 & -4.21420700 & -3.27748900 & 2.87687400 & $\mathrm{H}$ & 0 & -1.32805300 & -4.13434800 & 3.7444690 \\
\hline $\mathrm{C}$ & -1 & 3.24266100 & -0.92537300 & -0.09652800 & $\mathrm{H}$ & 0 & 0.06603000 & -3.64813500 & 2.76060300 \\
\hline $\mathrm{C}$ & -1 & 5.44588100 & -2.33439900 & -0.04436600 & $\mathrm{H}$ & 0 & -0.02068600 & -3.17223200 & 4.4580090 \\
\hline $\mathrm{C}$ & -1 & -1.57672500 & -2.31756200 & 0.82754700 & $\mathrm{H}$ & 0 & -4.07089500 & -2.03565200 & 1.08649200 \\
\hline $\mathrm{C}$ & -1 & -0.40676400 & -2.42936100 & 0.14018400 & $\mathrm{H}$ & 0 & 7.43266800 & -3.12245600 & 0.06800100 \\
\hline $\mathrm{C}$ & -1 & -1.58048500 & -2.48438500 & -0.55888000 & $\mathrm{H}$ & 0 & -3.95501000 & -4.20714000 & 2.35717500 \\
\hline $\mathrm{C}$ & -1 & -4.00103100 & -1.28522900 & -2.69703600 & $\mathrm{H}$ & 0 & -3.79748900 & -3.31672300 & 3.89044900 \\
\hline C & -1 & -0.35044800 & -1.57270300 & -2.98975900 & $\mathrm{H}$ & 0 & -5.30637300 & -3.21721300 & 2.96489200 \\
\hline $\mathrm{C}$ & -1 & -3.64400800 & -2.49821400 & -1.86312100 & $\mathrm{H}$ & 0 & 4.95405200 & -3.30168100 & -0.10156100 \\
\hline $\mathrm{C}$ & -1 & -1.33316900 & -2.69348400 & -2.91559300 & $\mathrm{H}$ & 0 & -3.54466100 & -0.38097400 & -2.28011700 \\
\hline $\mathrm{C}$ & -1 & -4.21767900 & -3.78181300 & -2.43726200 & $\mathrm{H}$ & 0 & -3.68127300 & -1.38592200 & -3.74074000 \\
\hline $\mathrm{C}$ & -1 & -0.65613600 & -4.06484800 & -2.95434700 & $\mathrm{H}$ & 0 & -5.08981800 & -1.14379500 & -2.71192800 \\
\hline $\mathrm{N}$ & -1 & -0.51226000 & 2.30784500 & -0.09307500 & $\mathrm{H}$ & 0 & -0.83369800 & -0.59467000 & -2.88757800 \\
\hline $\mathrm{N}$ & -1 & 3.02838400 & 0.43626000 & -0.06220700 & $\mathrm{H}$ & 0 & 0.42495000 & -1.62977000 & -2.22017100 \\
\hline $\mathrm{N}$ & -1 & -2.19228800 & -2.19247700 & 1.96216600 & $\mathrm{H}$ & 0 & 0.16282300 & -1.59225000 & -3.96030300 \\
\hline $\mathrm{N}$ & -1 & -2.19599500 & -2.60155200 & -1.70224100 & $\mathrm{H}$ & 0 & -4.05863200 & -2.35284000 & -0.85888200 \\
\hline $\mathrm{O}$ & -1 & 0.14144500 & 4.51975100 & -0.01318400 & $\mathrm{H}$ & 0 & -2.01062400 & -2.62976900 & -3.77344600 \\
\hline $\mathrm{O}$ & -1 & 4.40181800 & 2.29121600 & 0.04728700 & $\mathrm{H}$ & 0 & -3.97189400 & -4.64891800 & -1.81189600 \\
\hline $\mathrm{O}$ & -1 & -1.88569500 & 0.45288900 & -0.20255200 & $\mathrm{H}$ & 0 & -5.31079700 & -3.71297700 & -2.50650600 \\
\hline $\mathrm{O}$ & -1 & 2.37358400 & -1.77619200 & -0.14146500 & $\mathrm{H}$ & 0 & -3.84206600 & -3.97704600 & -3.44939000 \\
\hline $\mathrm{Au}$ & -1 & 1.25811300 & 1.37239300 & -0.07727000 & $\mathrm{H}$ & 0 & -1.39064600 & -4.87879800 & -2.93440700 \\
\hline $\mathrm{H}$ & 0 & 0.67129200 & -2.31789800 & 0.10878800 & $\mathrm{H}$ & 0 & -0.05909400 & -4.16765000 & -3.86938700 \\
\hline $\mathrm{H}$ & 0 & -2.43413900 & 6.04249500 & -0.05464000 & $\mathrm{H}$ & 0 & 0.02544500 & -4.19528100 & -2.10538300 \\
\hline
\end{tabular}

\section{Optimized structure of $\mathrm{AuBF}_{4}$}

\# opt=calcfc freq=noraman b3lyp/lanl2dz

\section{Zero-point correction=}

Thermal correction to Energy=

Thermal correction to Enthalpy=
0.013835 (Hartree/Particle)

0.020671

0.021615 


\begin{tabular}{|c|c|}
\hline Thermal correction to Gibbs Free Energy= & -0.020493 \\
\hline Sum of electronic and zero-point Energies= & -559.858996 \\
\hline Sum of electronic and thermal Energies= & -559.852160 \\
\hline Sum of electronic and thermal Enthalpies $=$ & -559.851216 \\
\hline Sum of electronic and thermal Free Energies= & -559.893323 \\
\hline \multicolumn{2}{|l|}{01} \\
\hline $\begin{array}{lll}-2.01556100 & 0.00002800 & 0.00006600\end{array}$ & \\
\hline $\begin{array}{lll}-1.03037400 & 0.00037900 & -1.15678100\end{array}$ & \\
\hline $\begin{array}{lll}-2.73467400 & 1.18699700 & 0.00073900\end{array}$ & \\
\hline $\begin{array}{lll}-1.03061700 & -0.00129800 & 1.15687900\end{array}$ & \\
\hline$-2.73600000-1.18608900-0.00089700$ & \\
\hline $\begin{array}{lll}0.98560500 & -0.00000100 & 0.00000300\end{array}$ & \\
\hline
\end{tabular}

\section{Optimized structure of 11}

\# opt=calcfc freq=noraman b3lyp/lanl2dz

Zero-point correction $=$

Thermal correction to Energy=
0.585640 (Hartree/Particle)

0.623089

Thermal correction to Enthalpy= $\quad 0.624033$

Thermal correction to Gibbs Free Energy= 0.513523

Sum of electronic and zero-point Energies $=\quad-1482.429394$

Sum of electronic and thermal Energies= $\quad-1482.391945$

Sum of electronic and thermal Enthalpies $=\quad-1482.391001$

Sum of electronic and thermal Free Energies $=\quad-1482.501511$

01

$\begin{array}{llll}\mathrm{N} & -0.72437600 & -0.29206400 & 0.99264700\end{array}$

$\begin{array}{llll}\mathrm{N} & 1.95082000 & 2.07267100 & 0.27307200\end{array}$

$\mathrm{N} \quad 2.60871100 \quad-1.60590400 \quad-0.21942800$

$\begin{array}{llll}\mathrm{N} & -2.02316500 & -1.67288600 & -0.54727600\end{array}$

$\mathrm{N} \quad \begin{array}{llll}\mathrm{N} & -2.67240600 & -1.46000800 & 1.59532600\end{array}$

$\begin{array}{llll}\mathrm{C} & 0.48101700 & -0.12947500 & 0.47473300\end{array}$

$\begin{array}{llll}\text { C } & 1.53224600 & 0.79816800 & 0.26458200\end{array}$

$\begin{array}{llll}\text { C } & 1.76297300 & -0.58790600 & 0.05949900\end{array}$

$\begin{array}{llll}\text { C } & -1.72605400 & -1.10495100 & 0.67048600\end{array}$

$\begin{array}{llll}\text { C } & -3.32701600 & -2.38874800 & -0.49152400\end{array}$

$\mathrm{H} \quad-3.17780900 \quad-3.45968400 \quad-0.68077300$

$\mathrm{H} \quad-4.00278600 \quad-1.96593300 \quad-1.23958500$

$\begin{array}{llll}\text { C } & -3.83616500 & -2.10628300 & 0.94907600\end{array}$

$\begin{array}{llll}\mathrm{H} & -4.68238500 & -1.41089600 & 0.94117000\end{array}$
$\mathrm{H} \quad-4.11214600 \quad-3.02315200 \quad 1.48252700$

C $\quad-1.49990400 \quad-1.25581400 \quad-1.85326600$

$\mathrm{H} \quad-1.18088100 \quad-2.13348200 \quad-2.43160400$

$\mathrm{H} \quad-2.27652900 \quad-0.71082400 \quad-2.39955700$

$\mathrm{H} \quad-0.64859100 \quad-0.58516900 \quad-1.72721600$

$\begin{array}{llll}\text { C } & -2.82086900 & -0.79358000 & 2.88750600\end{array}$

$\begin{array}{llll}\mathrm{H} & -3.56506900 & 0.01032500 & 2.80825400\end{array}$

$\mathrm{H} \quad-3.13534900 \quad-1.51705800 \quad 3.65004800$

$\mathrm{H} \quad-1.86134600 \quad-0.35840800 \quad 3.17515600$

$\begin{array}{llll}\text { C } & 0.95131800 & 3.18349800 & 0.48282300\end{array}$

$\begin{array}{llll}\mathrm{H} & 1.55615700 & 4.09611400 & 0.54494000\end{array}$

$\begin{array}{lllll}\text { C } & 0.19089900 & 3.00990900 & 1.81507500\end{array}$

$\begin{array}{lllll}\mathrm{H} & -0.46450500 & 3.87466300 & 1.97604000\end{array}$

$\begin{array}{llll}\mathrm{H} & -0.44173000 & 2.11582700 & 1.79305400\end{array}$

$\begin{array}{llll}\mathrm{H} & 0.88448500 & 2.93817500 & 2.66327100\end{array}$ 


$\begin{array}{llll}\mathrm{C} & -0.01252400 & 3.31180300 & -0.71498100 \\ \mathrm{H} & -0.63862100 & 4.20448300 & -0.58956800 \\ \mathrm{H} & 0.53918000 & 3.41325300 & -1.65822700 \\ \mathrm{H} & -0.69699600 & 2.46130700 & -0.78938300 \\ \mathrm{C} & 3.39421000 & 2.40684100 & 0.07894900 \\ \mathrm{H} & 3.90932600 & 1.44594300 & -0.03762100 \\ \mathrm{C} & 3.62418000 & 3.23374200 & -1.20554200 \\ \mathrm{H} & 4.69737500 & 3.41290700 & -1.34972300 \\ \mathrm{H} & 3.23787800 & 2.70875700 & -2.08716700 \\ \mathrm{H} & 3.12806200 & 4.20989900 & -1.14843000 \\ \mathrm{C} & 3.98920700 & 3.09703200 & 1.32795500 \\ \mathrm{H} & 5.06791100 & 3.24715900 & 1.19236000 \\ \mathrm{H} & 3.53750100 & 4.08077300 & 1.50298800 \\ \mathrm{H} & 3.83441800 & 2.48461500 & 2.22411000 \\ \mathrm{C} & 2.25261400 & -3.00526200 & 0.18335600 \\ \mathrm{H} & 3.16222700 & -3.59376800 & 0.01535600 \\ \mathrm{C} & 1.90850200 & -3.08746900 & 1.68742300 \\ \mathrm{H} & 1.73689700 & -4.13270300 & 1.97356500 \\ \mathrm{H} & 2.72701900 & -2.69215800 & 2.30139700 \\ \mathrm{H} & 0.99802300 & -2.52353800 & 1.92138100\end{array}$

$\begin{array}{lrrr}\text { C } & 1.12953900 & -3.59983500 & -0.69374700 \\ \mathrm{H} & 0.97690200 & -4.65620600 & -0.43661000 \\ \mathrm{H} & 0.18088200 & -3.07641100 & -0.53369700 \\ \mathrm{H} & 1.38661200 & -3.53921300 & -1.75765600 \\ \mathrm{C} & 3.94090600 & -1.33370600 & -0.84076500 \\ \mathrm{H} & 3.91810500 & -0.27804900 & -1.13615700 \\ \mathrm{C} & 4.15209700 & -2.15901800 & -2.12957800 \\ \mathrm{H} & 5.09868700 & -1.86930900 & -2.60287700 \\ \mathrm{H} & 4.20276100 & -3.23512700 & -1.92338000 \\ \mathrm{H} & 3.34120700 & -1.98144000 & -2.84521100 \\ \mathrm{C} & 5.09585200 & -1.53128400 & 0.16816700 \\ \mathrm{H} & 6.05394500 & -1.26020200 & -0.29336900 \\ \mathrm{H} & 4.95189200 & -0.90684300 & 1.05840800 \\ \mathrm{H} & 5.16898900 & -2.57665400 & 0.49367200 \\ \mathrm{~B} & -4.02823100 & 1.28500700 & -0.91658800 \\ \mathrm{~F} & -4.46952300 & 0.18385800 & -1.75429300 \\ \mathrm{~F} & -4.78636900 & 2.45339300 & -1.17228700 \\ \mathrm{~F} & -2.62091300 & 1.52006700 & -1.19355700 \\ \mathrm{~F} & -4.16161700 & 0.87805200 & 0.47028100\end{array}$

\section{Optimized structure of 10}

\# opt=calcfc freq=noraman b3lyp/lanl2dz

Zero-point correction $=$

Thermal correction to Energy=

Thermal correction to Enthalpy=
0.602113 (Hartree/Particle)

\author{
0.647660
}

0.648604

0.518530

$-2042.379754$

$-2042.334206$

$-2042.333262$

\footnotetext{
Sum of electronic and thermal Enthalpies=

Sum of electronic and thermal Free Energies=

01

$\begin{array}{llll}\mathrm{N} & 0.54268100 & 0.40169400 & -0.18119600\end{array}$

$\mathrm{N} \quad \begin{array}{llll}\mathrm{N} & -2.26206100 & -1.91434300 & -0.88357000\end{array}$

$\begin{array}{llll}\mathrm{N} & -2.93022700 & 1.75172600 & -0.85556000\end{array}$

$\begin{array}{llll}\mathrm{N} & 1.81246600 & 2.32202600 & -0.93679000\end{array}$

$\begin{array}{llll}\mathrm{N} & 1.42674400 & 2.12663700 & 1.26039600\end{array}$

$\begin{array}{llll}\text { C } & -0.75926300 & 0.23963400 & -0.51417400\end{array}$
}

$-2042.463336$

$\begin{array}{llll}\text { C } & -1.80413900 & -0.66364700 & -0.76890400\end{array}$

$\begin{array}{lllll}\text { C } & -2.05644100 & 0.73676000 & -0.75052600\end{array}$

$\begin{array}{llll}\mathrm{C} & 1.19311300 & 1.60851000 & 0.03912100\end{array}$

$\begin{array}{llll}\text { C } & 2.70598000 & 3.35308800 & -0.34330600\end{array}$

$\begin{array}{llll}\mathrm{H} & 2.46468800 & 4.34348300 & -0.74265700\end{array}$

$\begin{array}{llll}\mathrm{H} & 3.74694400 & 3.10906600 & -0.58082700\end{array}$

$\begin{array}{llll}\text { C } & 2.41884200 & 3.23235900 & 1.18390000\end{array}$ 


\begin{tabular}{|c|c|c|c|c|c|c|c|}
\hline $\mathrm{H}$ & 3.31570600 & 2.95025600 & 1.74450200 & $\mathrm{H}$ & -3.55613200 & 3.67436500 & -0.41598500 \\
\hline $\mathrm{H}$ & 1.99500600 & 4.14752900 & 1.61006700 & $\mathrm{C}$ & -2.24443400 & 3.08007300 & 1.17049700 \\
\hline $\mathrm{C}$ & 1.98456500 & 1.88865800 & -2.32895000 & $\mathrm{H}$ & -2.14869500 & 4.10504500 & 1.55224600 \\
\hline $\mathrm{H}$ & 1.94533700 & 2.76192800 & -2.98904600 & $\mathrm{H}$ & -3.00635600 & 2.55601300 & 1.75530000 \\
\hline $\mathrm{H}$ & 2.95061600 & 1.37892600 & -2.44607800 & $\mathrm{H}$ & -1.29307100 & 2.56622500 & 1.33798200 \\
\hline $\mathrm{H}$ & 1.18144100 & 1.20223400 & -2.60472700 & $\mathrm{C}$ & -1.56589200 & 3.84435300 & -1.19406000 \\
\hline $\mathrm{C}$ & 1.06145100 & 1.52137100 & 2.55085500 & $\mathrm{H}$ & -1.41833000 & 4.86688400 & -0.82241800 \\
\hline $\mathrm{H}$ & 1.91898900 & 0.96190500 & 2.94919900 & $\mathrm{H}$ & -0.59601200 & 3.33548600 & -1.16745900 \\
\hline $\mathrm{H}$ & 0.79529600 & 2.31794100 & 3.25425500 & $\mathrm{H}$ & -1.89307700 & 3.90301400 & -2.23886800 \\
\hline $\mathrm{H}$ & 0.20582800 & 0.85121900 & 2.44329000 & $\mathrm{C}$ & -4.30547200 & 1.49808400 & -1.40337700 \\
\hline $\mathrm{C}$ & -1.32367800 & -3.08480700 & -0.86880200 & $\mathrm{H}$ & -4.30008800 & 0.45523300 & -1.73492900 \\
\hline $\mathrm{H}$ & -1.95734600 & -3.95495200 & -1.07037700 & $\mathrm{C}$ & -4.57390000 & 2.37370300 & -2.64806000 \\
\hline $\mathrm{C}$ & -0.67106200 & -3.28209000 & 0.51667600 & $\mathrm{H}$ & -5.54844500 & 2.11000000 & -3.07756300 \\
\hline $\mathrm{H}$ & -0.08555900 & -4.21068800 & 0.52175800 & $\mathrm{H}$ & -4.60517400 & 3.44144800 & -2.39722600 \\
\hline $\mathrm{H}$ & 0.00183900 & -2.45129400 & 0.76393800 & $\mathrm{H}$ & -3.80557600 & 2.21985600 & -3.41570100 \\
\hline $\mathrm{H}$ & -1.42198300 & -3.32896900 & 1.31108700 & $\mathrm{C}$ & -5.40102200 & 1.65595600 & -0.32662200 \\
\hline $\mathrm{C}$ & -0.29299700 & -2.99605600 & -2.01384900 & $\mathrm{H}$ & -6.37020300 & 1.34800300 & -0.74047600 \\
\hline $\mathrm{H}$ & 0.32074300 & -3.90513900 & -2.03170300 & $\mathrm{H}$ & -5.17245200 & 1.03895800 & 0.54671300 \\
\hline $\mathrm{H}$ & -0.78992900 & -2.89427000 & -2.98669200 & $\mathrm{H}$ & -5.50251400 & 2.69990000 & -0.00261300 \\
\hline $\mathrm{H}$ & 0.38789900 & -2.14474200 & -1.88343700 & B & -2.75931200 & -0.53207700 & 2.69115900 \\
\hline $\mathrm{C}$ & -3.74564800 & -2.17155600 & -0.92466900 & $\mathrm{~F}$ & -2.96083400 & -1.89475000 & 3.06217300 \\
\hline $\mathrm{H}$ & -4.21252800 & -1.22541300 & -0.64423500 & $\mathrm{~F}$ & -1.38328900 & -0.36277900 & 2.22617000 \\
\hline $\mathrm{C}$ & -4.18506700 & -2.57310100 & -2.34993300 & $\mathrm{~F}$ & -3.00607900 & 0.37122900 & 3.75780900 \\
\hline $\mathrm{H}$ & -5.27240500 & -2.71776900 & -2.38180300 & $\mathrm{~F}$ & -3.63672200 & -0.21098600 & 1.56809000 \\
\hline $\mathrm{H}$ & -3.91692300 & -1.80330800 & -3.08543500 & $\mathrm{~B}$ & 5.03063100 & -0.33737000 & -0.14447300 \\
\hline $\mathrm{H}$ & -3.71636400 & -3.51620000 & -2.65954100 & $\mathrm{~F}$ & 4.48545300 & 0.62145400 & 0.78376700 \\
\hline $\mathrm{C}$ & -4.19273400 & -3.19815800 & 0.13729700 & $\mathrm{~F}$ & 6.34121800 & -0.70243100 & 0.12853300 \\
\hline $\mathrm{H}$ & -5.28960600 & -3.23666800 & 0.14411100 & $\mathrm{~F}$ & 4.79905100 & 0.11777600 & -1.47606700 \\
\hline $\mathrm{H}$ & -3.83253400 & -4.21176100 & -0.08016500 & $\mathrm{~F}$ & 4.15018400 & -1.60397400 & 0.01957800 \\
\hline $\mathrm{H}$ & -3.86094400 & -2.90049100 & 1.13553900 & $\mathrm{Au}$ & 2.07605800 & -0.96728600 & -0.05161400 \\
\hline $\mathrm{C}$ & -2.61741500 & 3.11778800 & -0.32634300 & & & & \\
\hline
\end{tabular}




\section{Spectra}

${ }^{1}$ H NMR [AuCl(BAC)] (8)

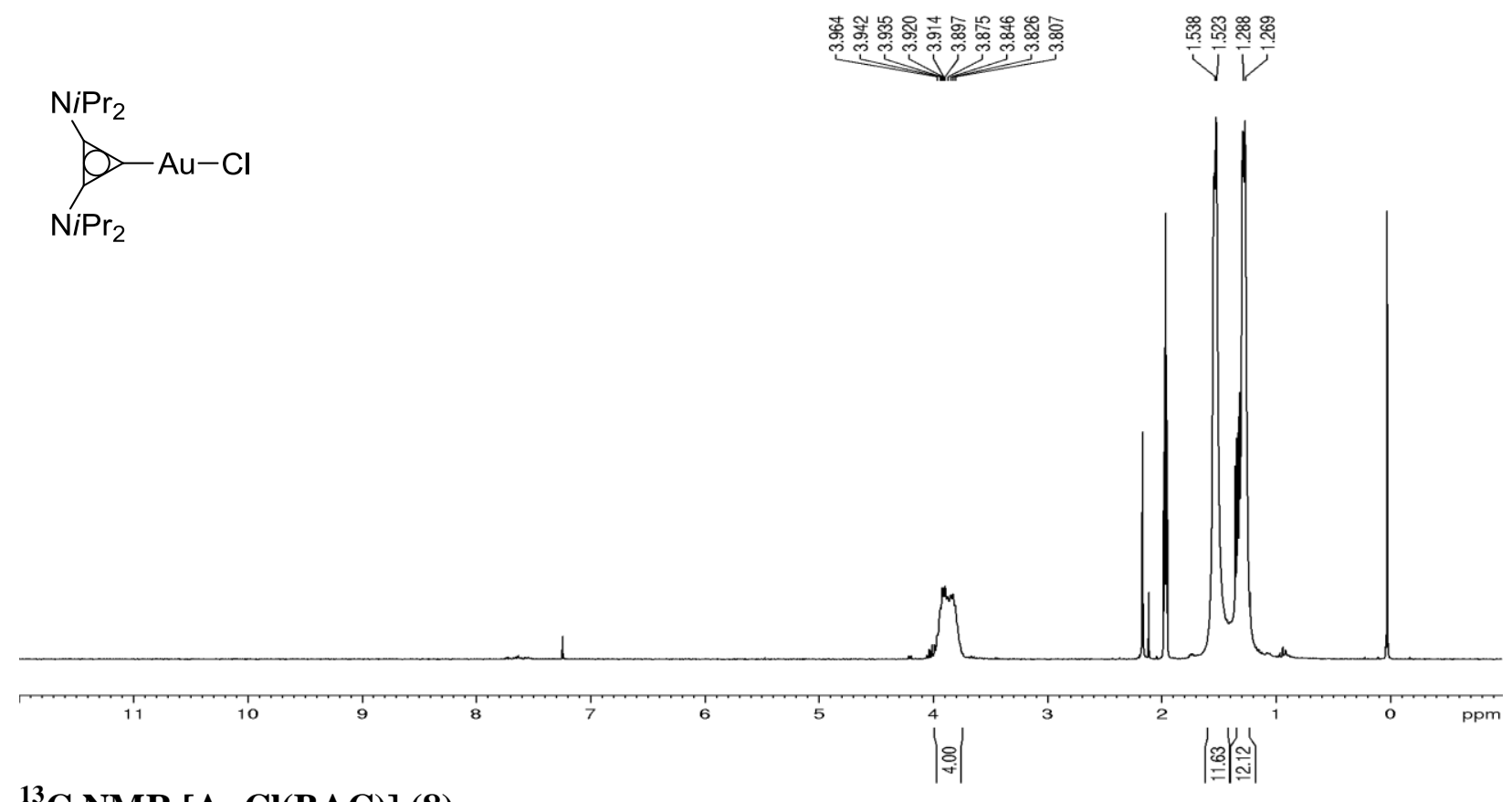

${ }^{13} \mathrm{C}$ NMR [AuCl(BAC)] (8)

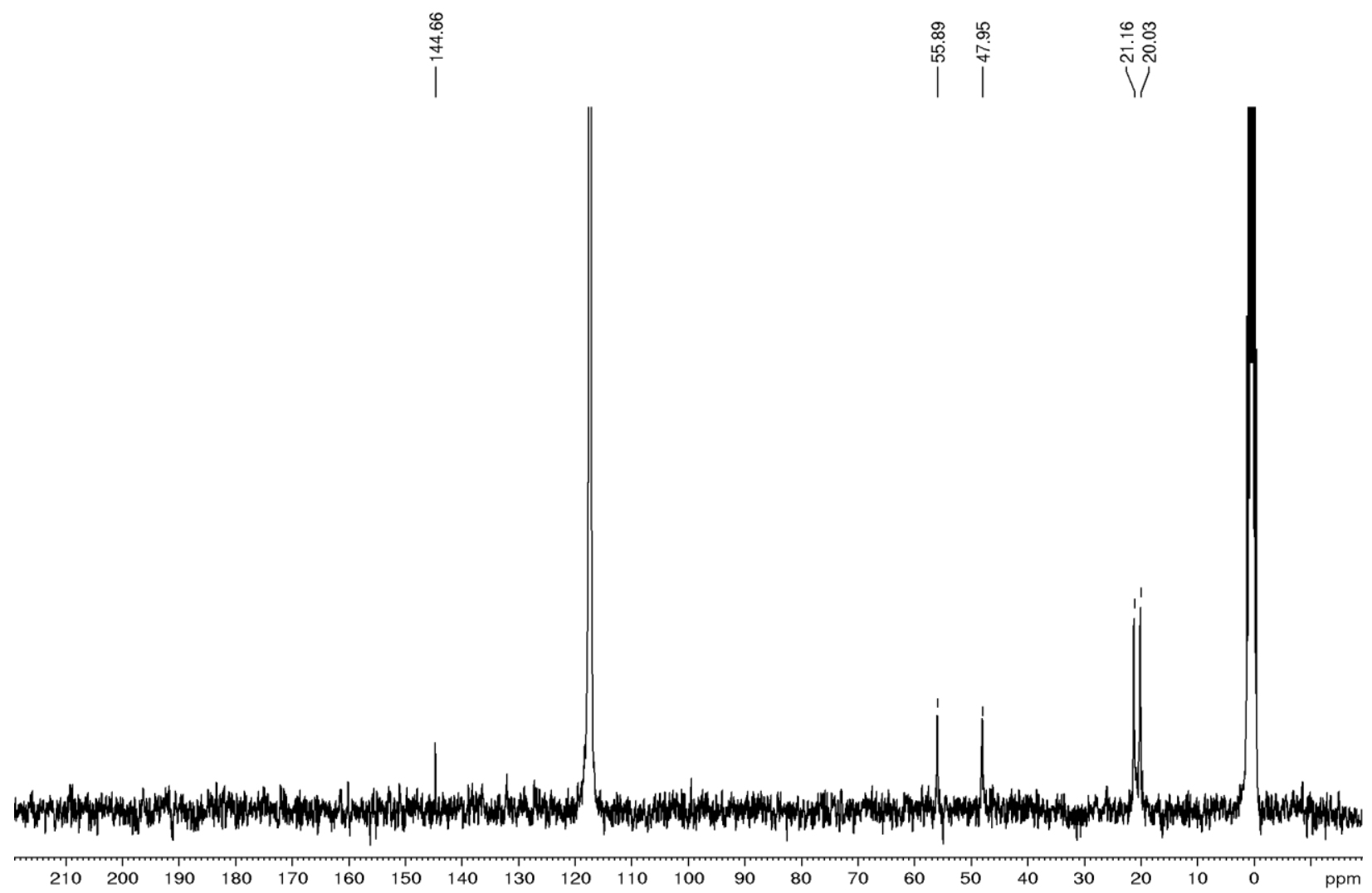


${ }^{1}$ H NMR Au(Pht) ${ }_{2}$ BACI-H (6)

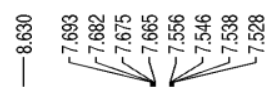

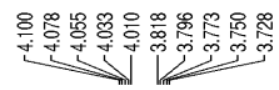

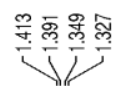<smiles>CCCNC1=Cc2ccccc2C1=O</smiles>

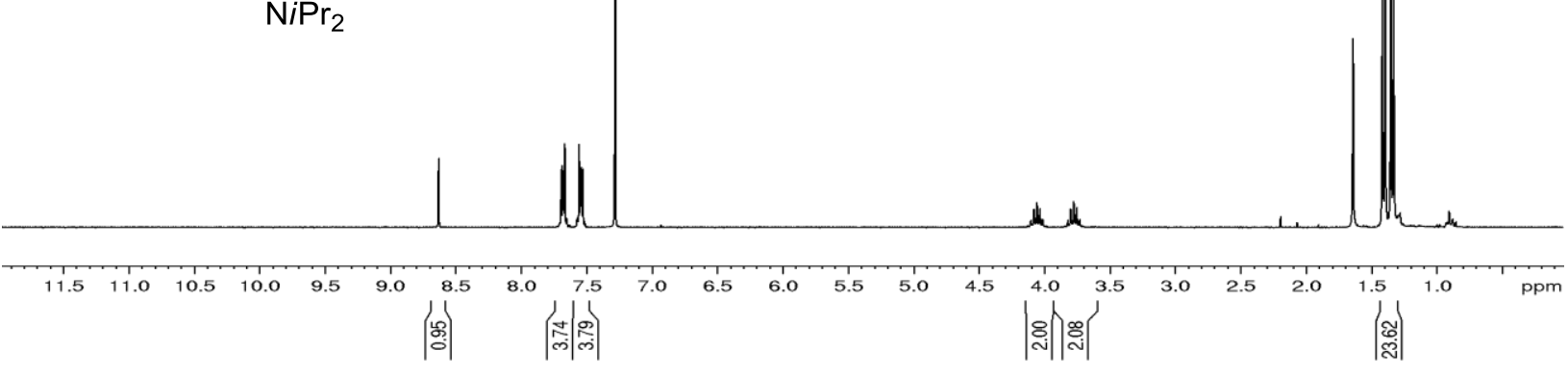

${ }^{13} \mathrm{C}$ NMR Au(Pht) $)_{2}$ BAC-H (6)

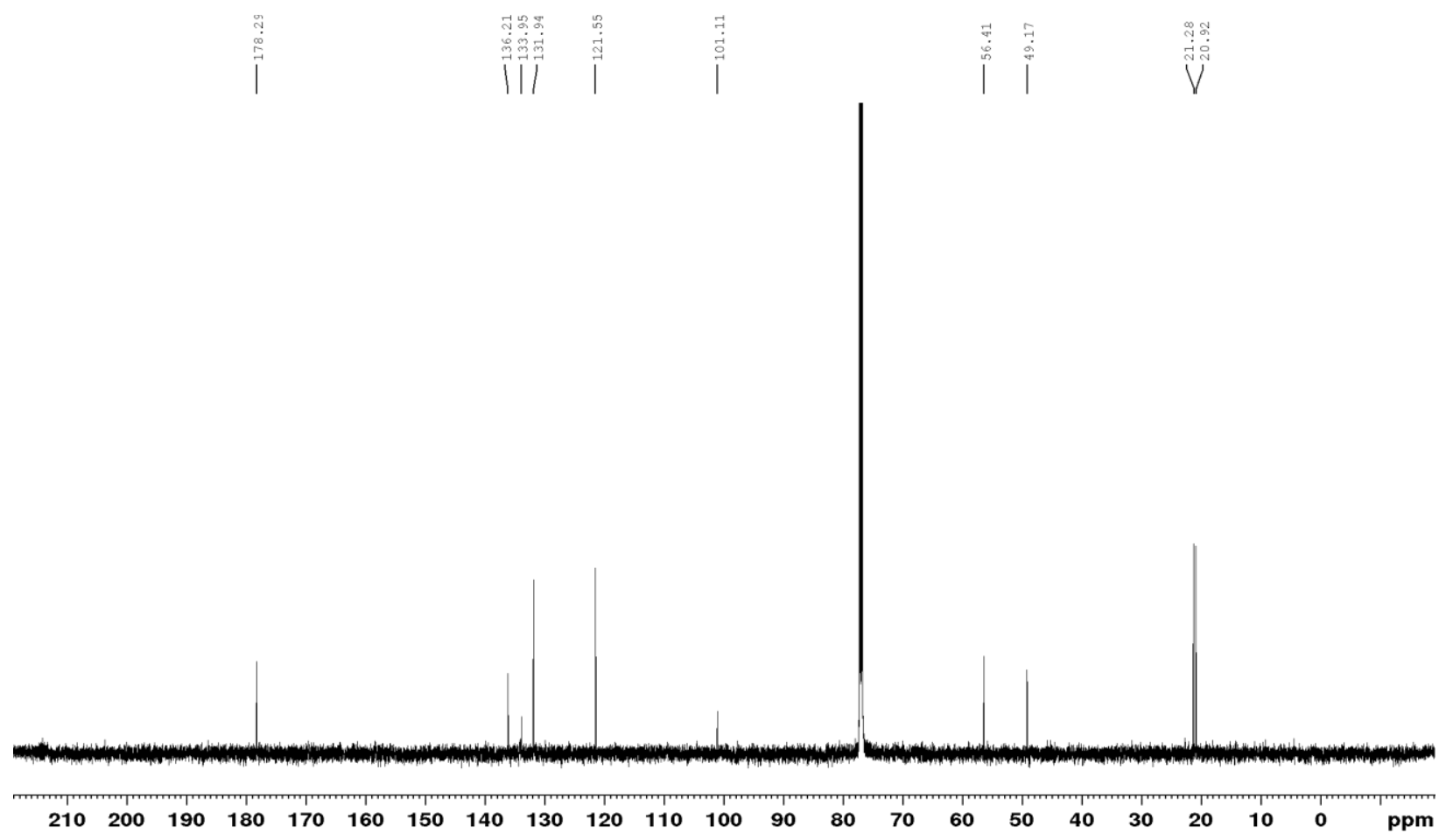

28 
IR Au(Pht) ${ }_{2}$ BACI-H (6)

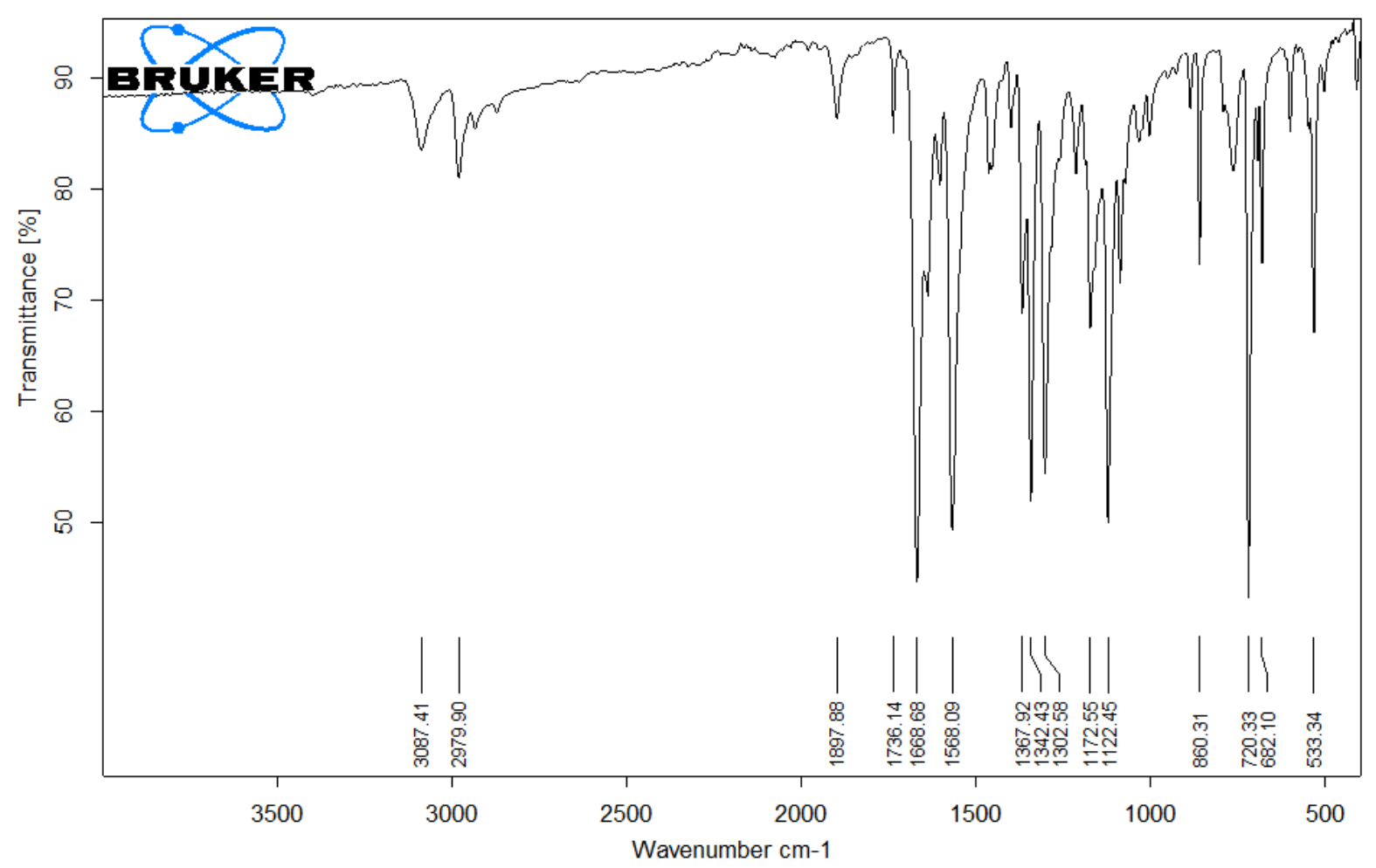




\section{${ }^{1}$ H NMR 5a}

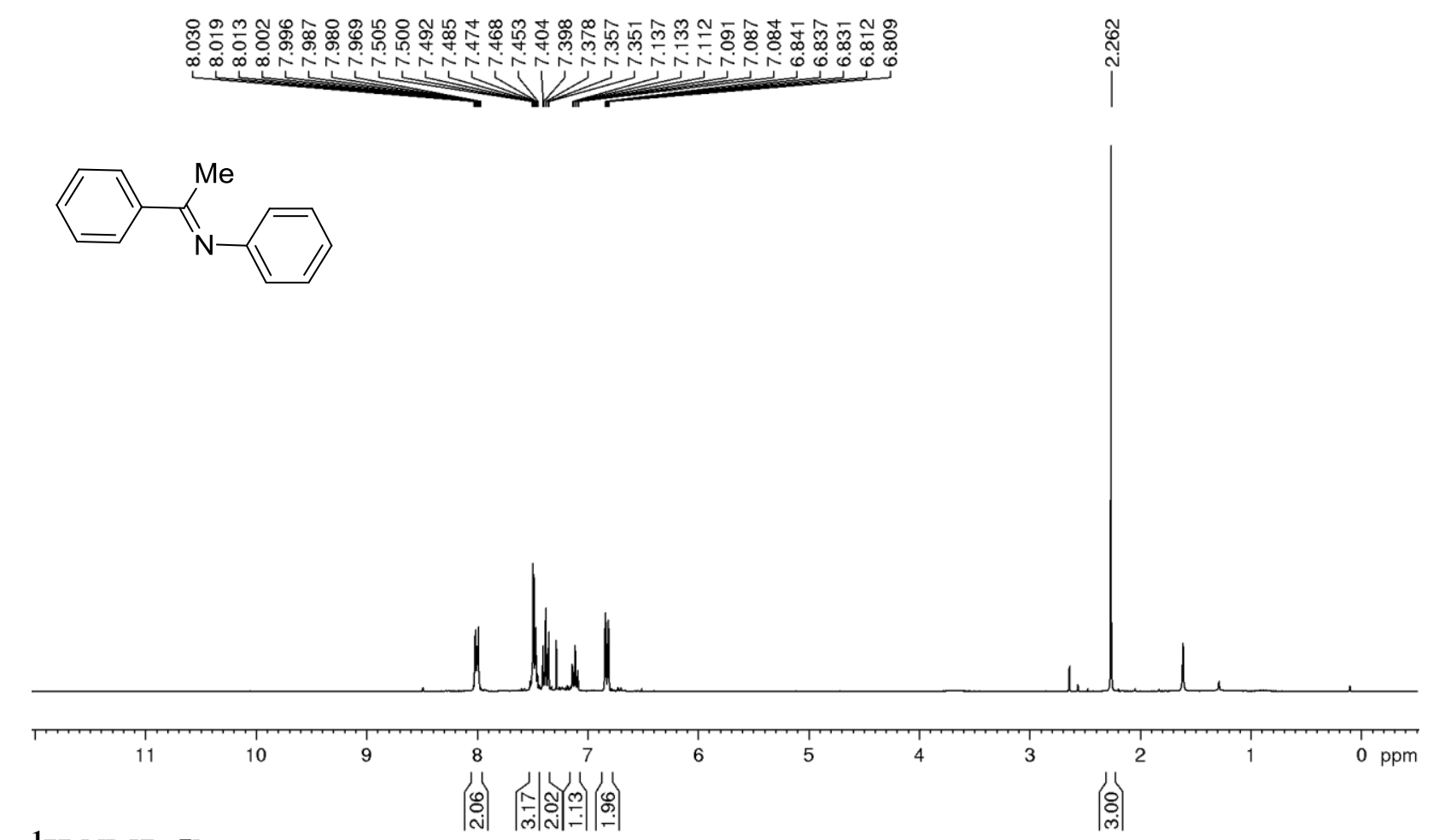

${ }^{1}$ H NMR 5b

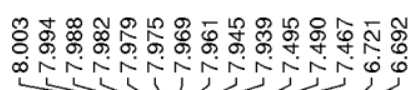
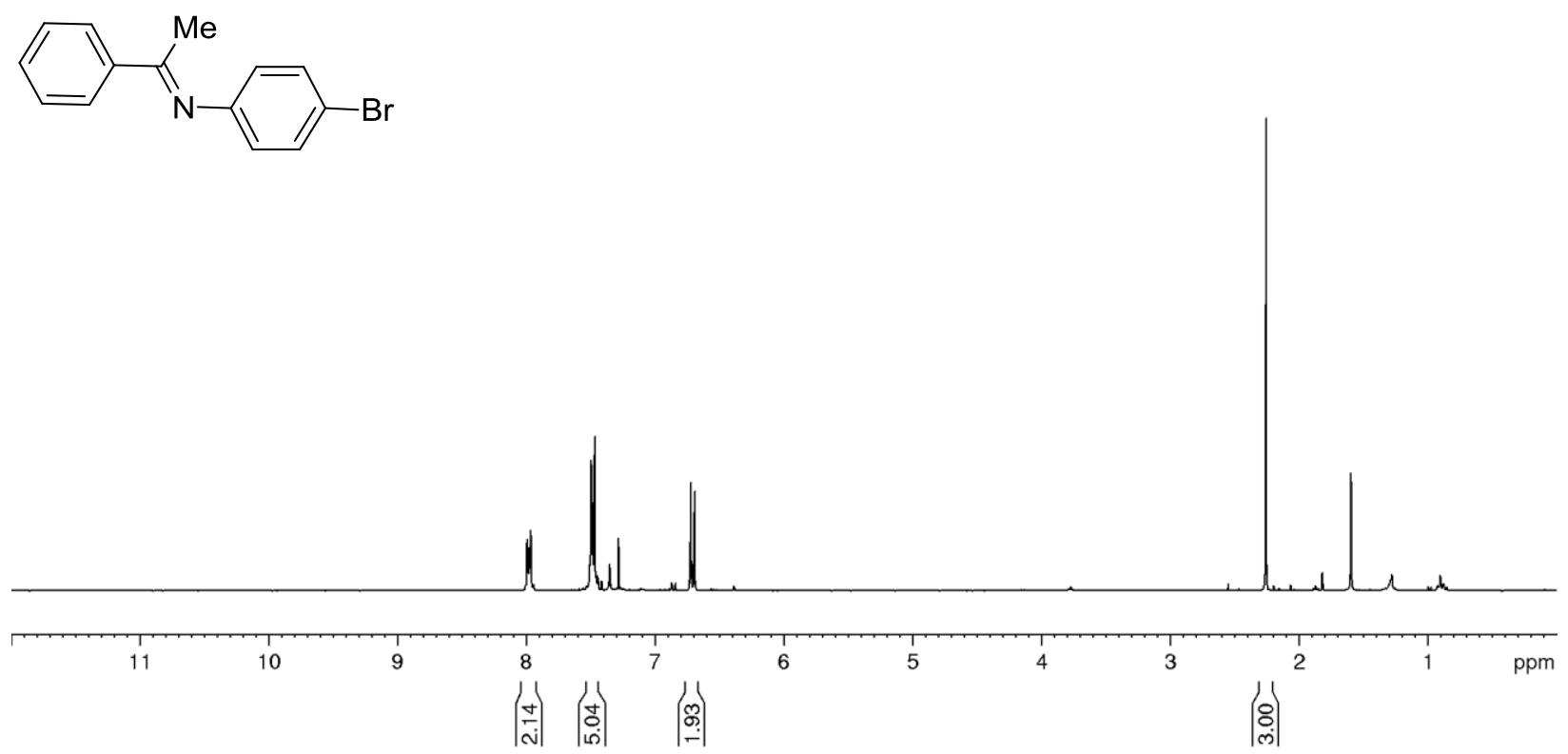


\section{${ }^{1}$ H NMR 5c}

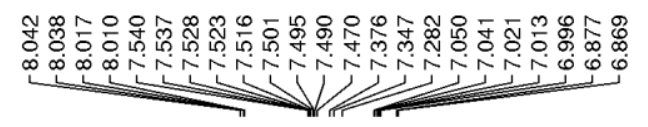<smiles>CC(=Nc1cc(Cl)ccc1Cl)c1ccccc1</smiles>

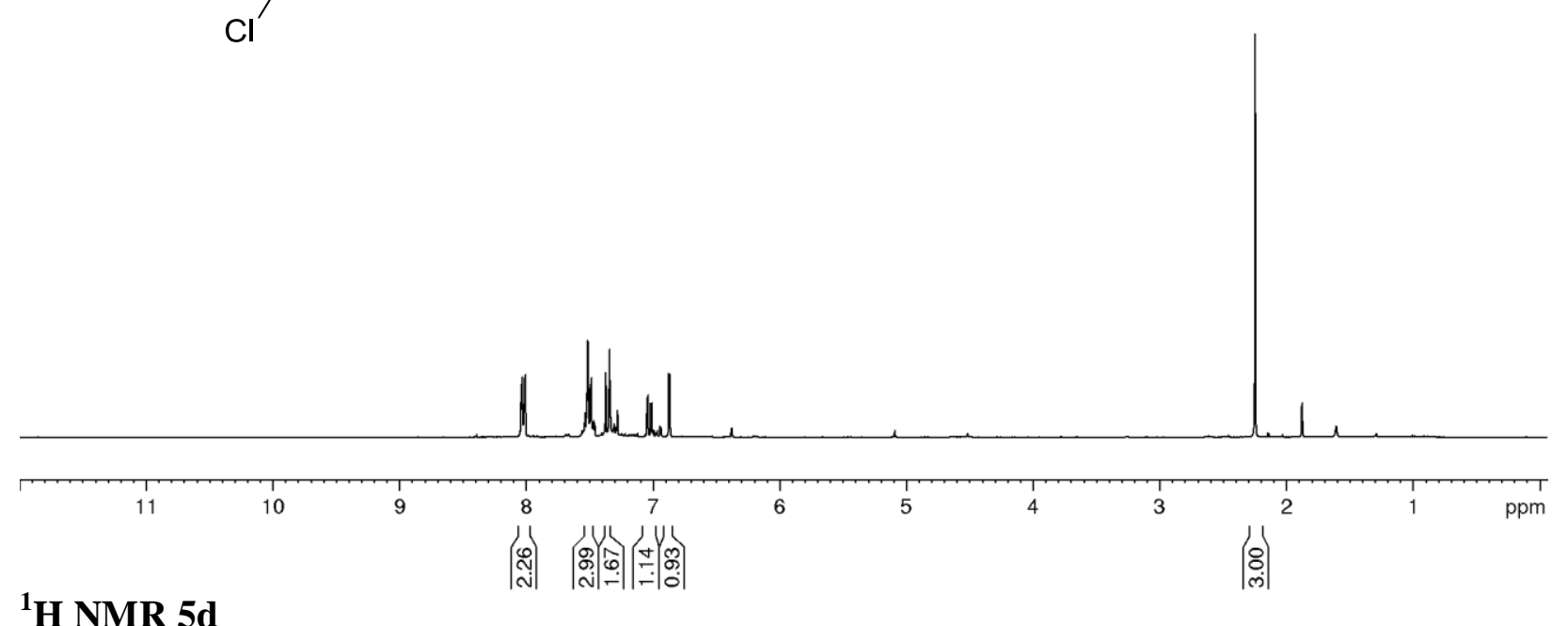

${ }^{1}$ H NMR 5d

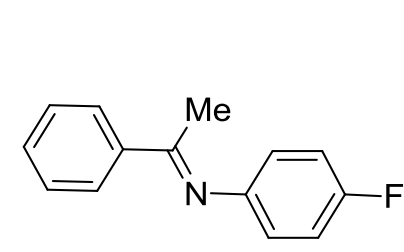

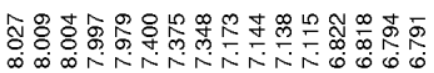

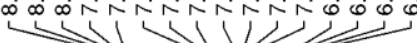

$\stackrel{ }{\stackrel{+}{N}}$

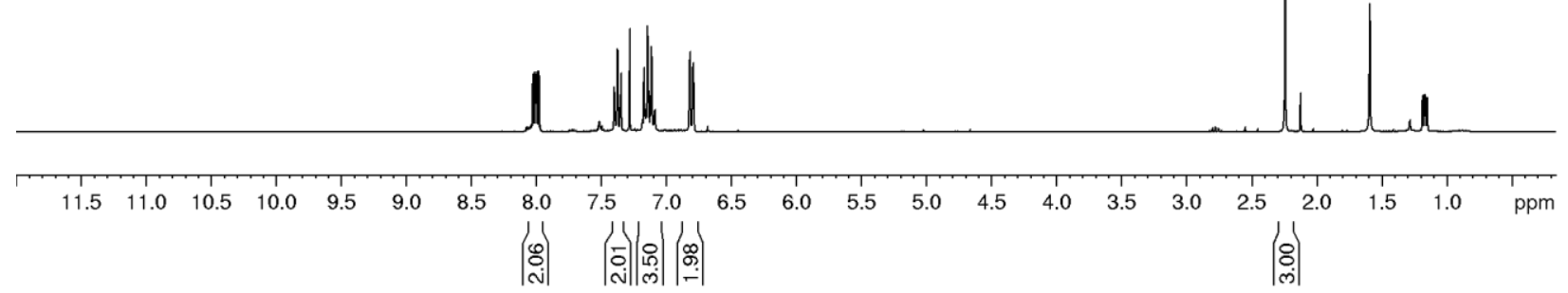




\section{${ }^{1}$ H NMR 5e}
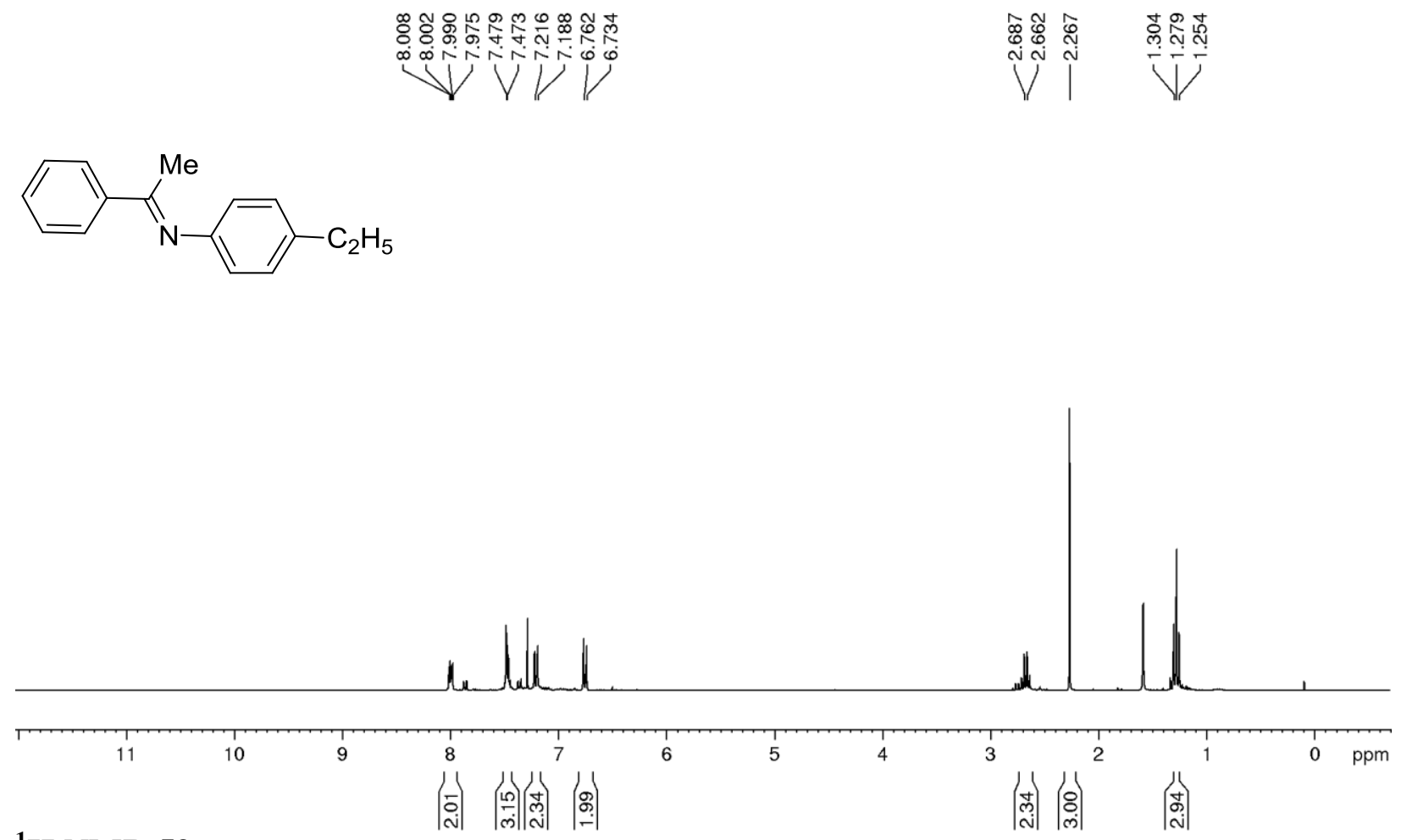

${ }^{1}$ H NMR 5f
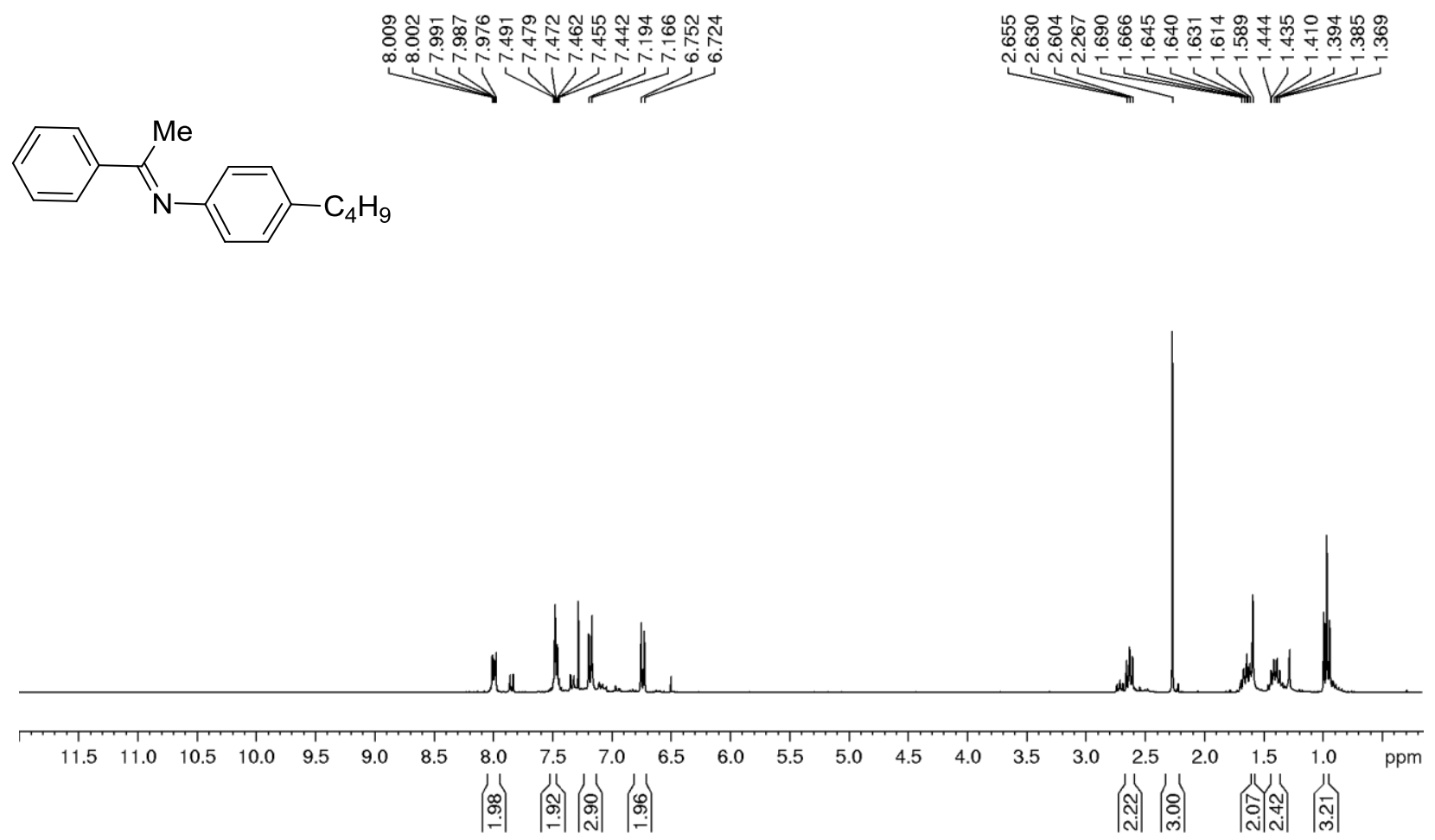


\section{${ }^{1}$ H NMR 5g}

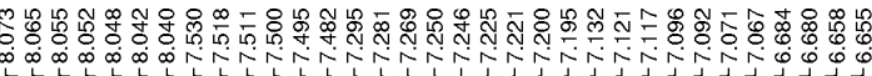

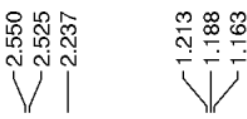

CON

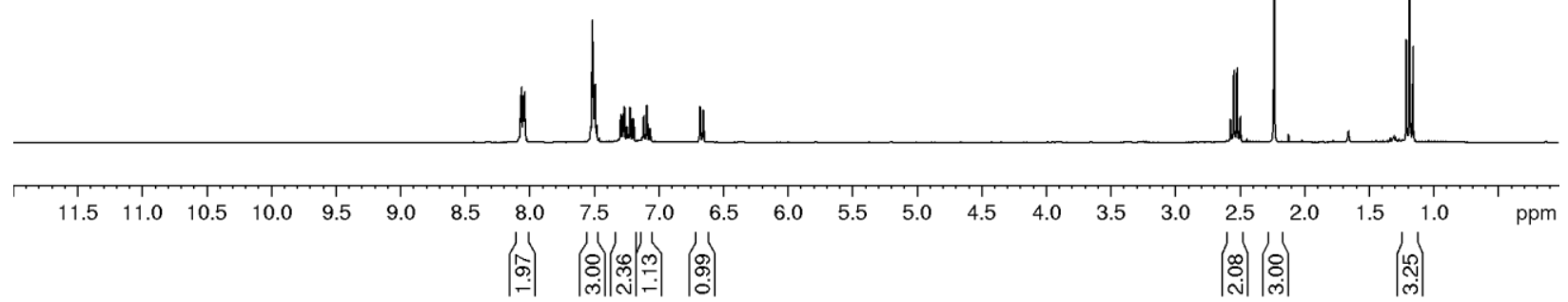

${ }^{1}$ H NMR 5 h

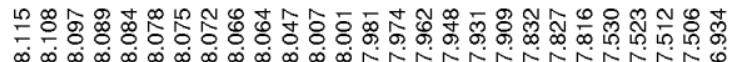

$\infty \infty_{\infty}^{\infty} \infty \infty_{\infty} \infty \infty_{\infty} \infty \infty_{\infty} \infty \infty^{\infty} \infty \infty^{\infty}$
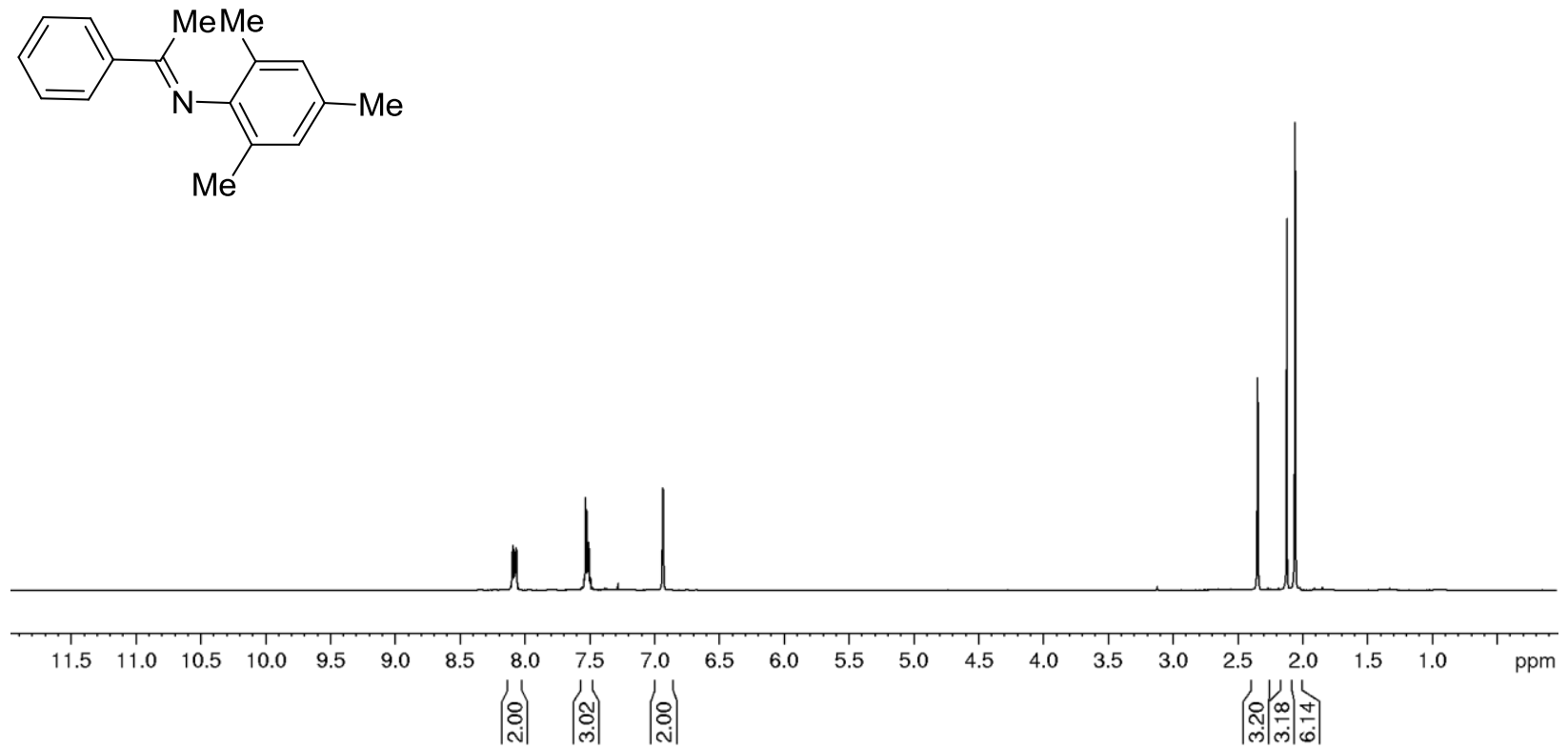


\section{${ }^{1}$ H NMR 5i}
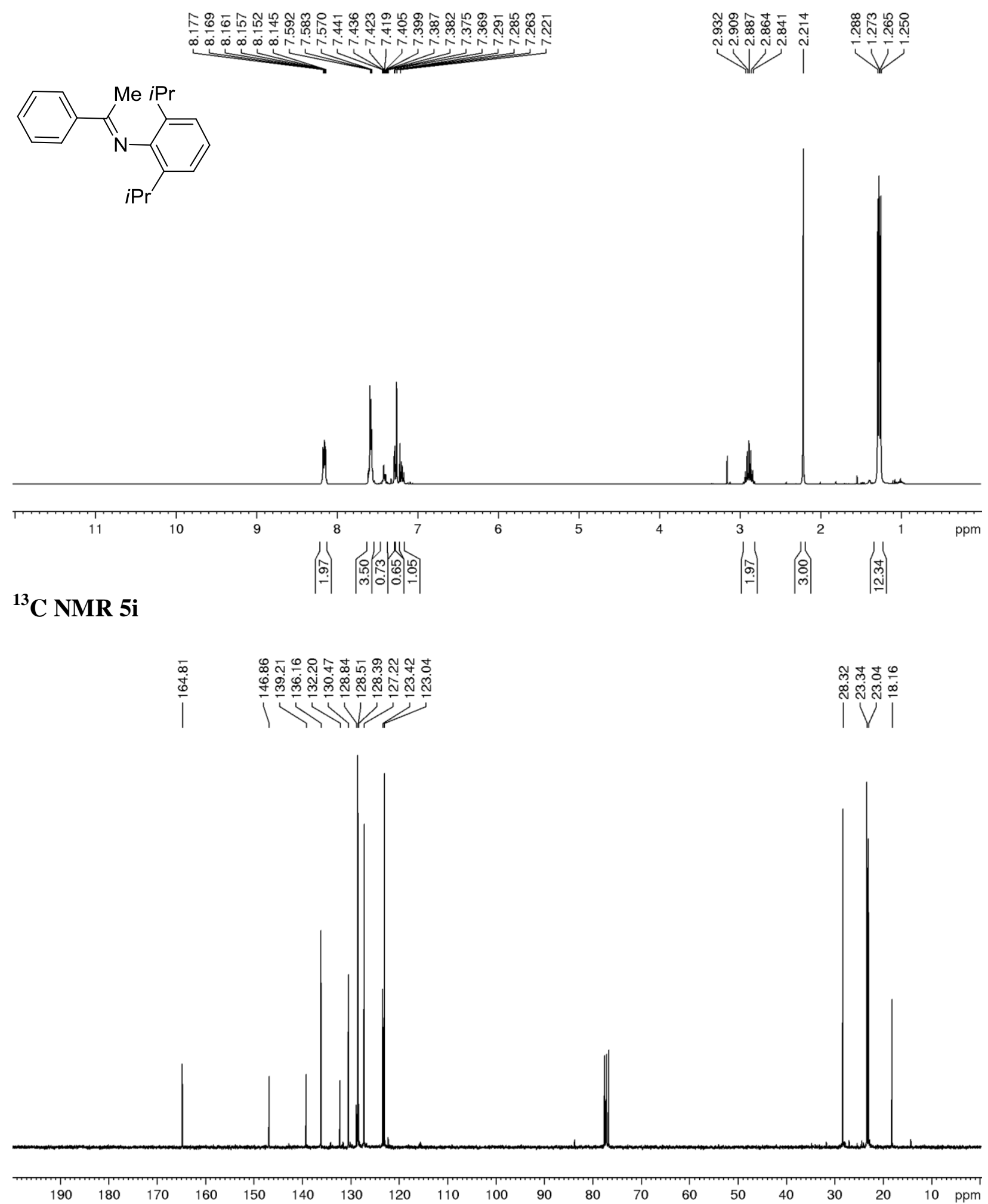


\section{${ }^{1}$ H NMR 5j}

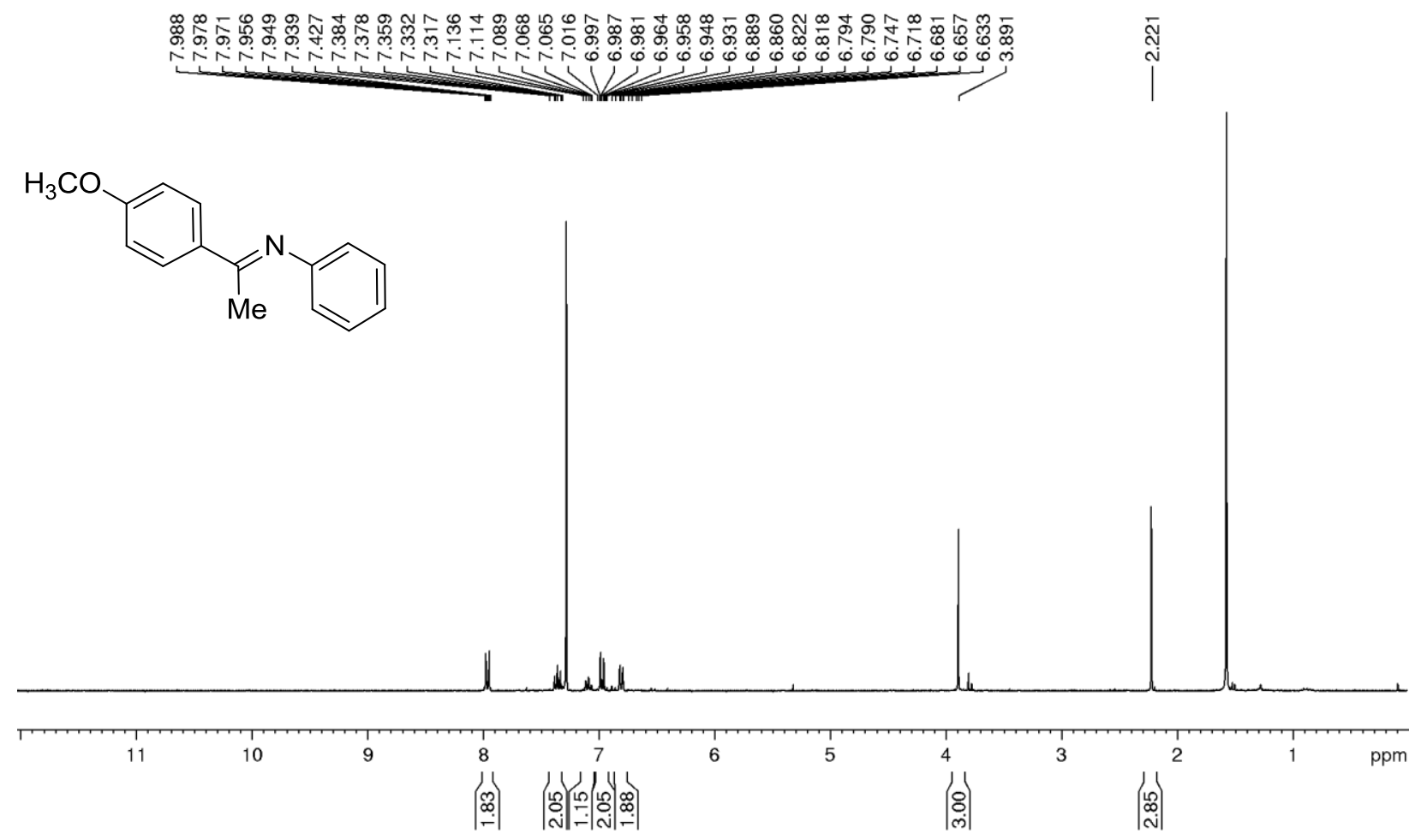

${ }^{1}$ H NMR 5k

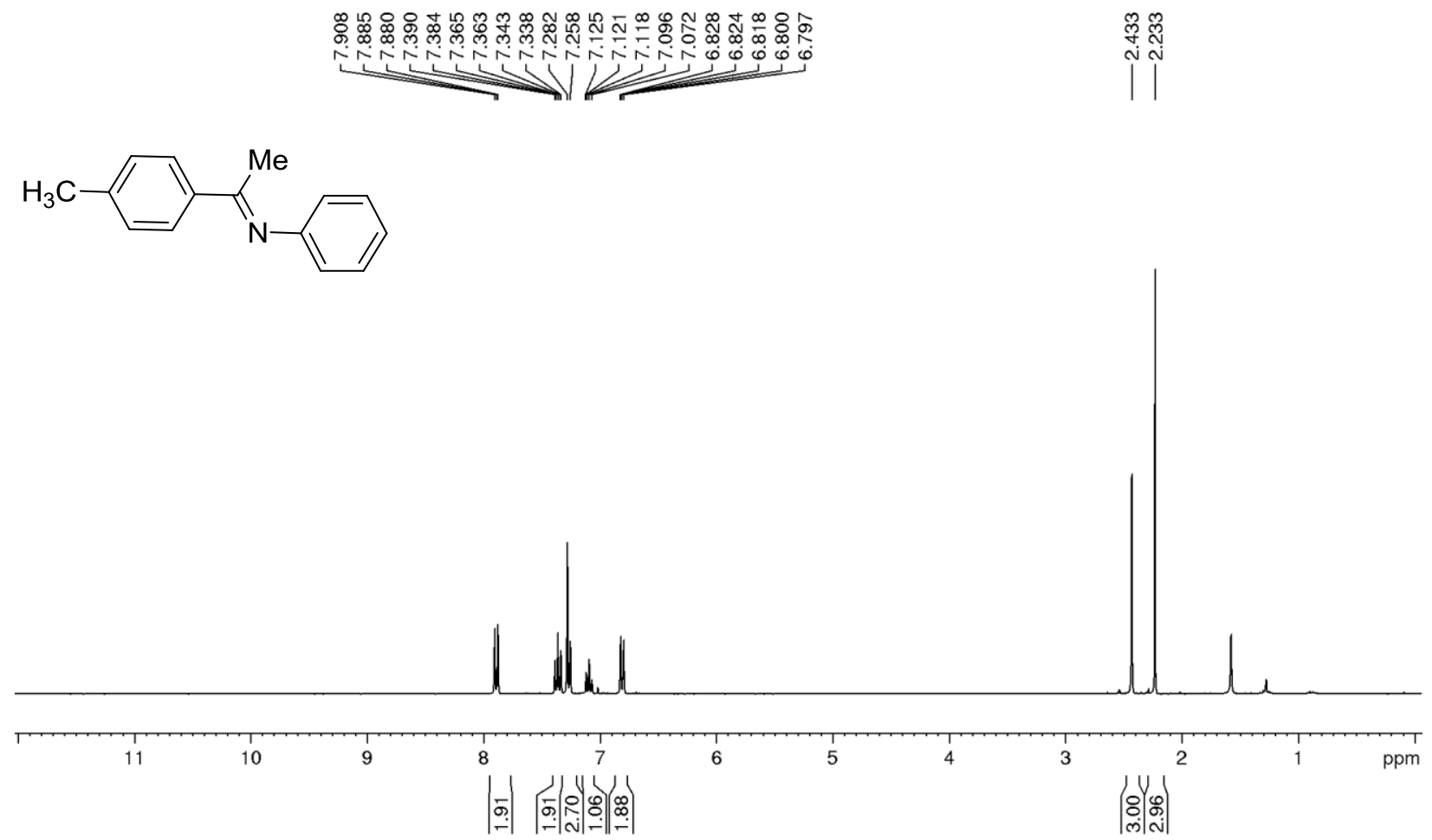




\section{${ }^{1}$ H NMR 5 I}

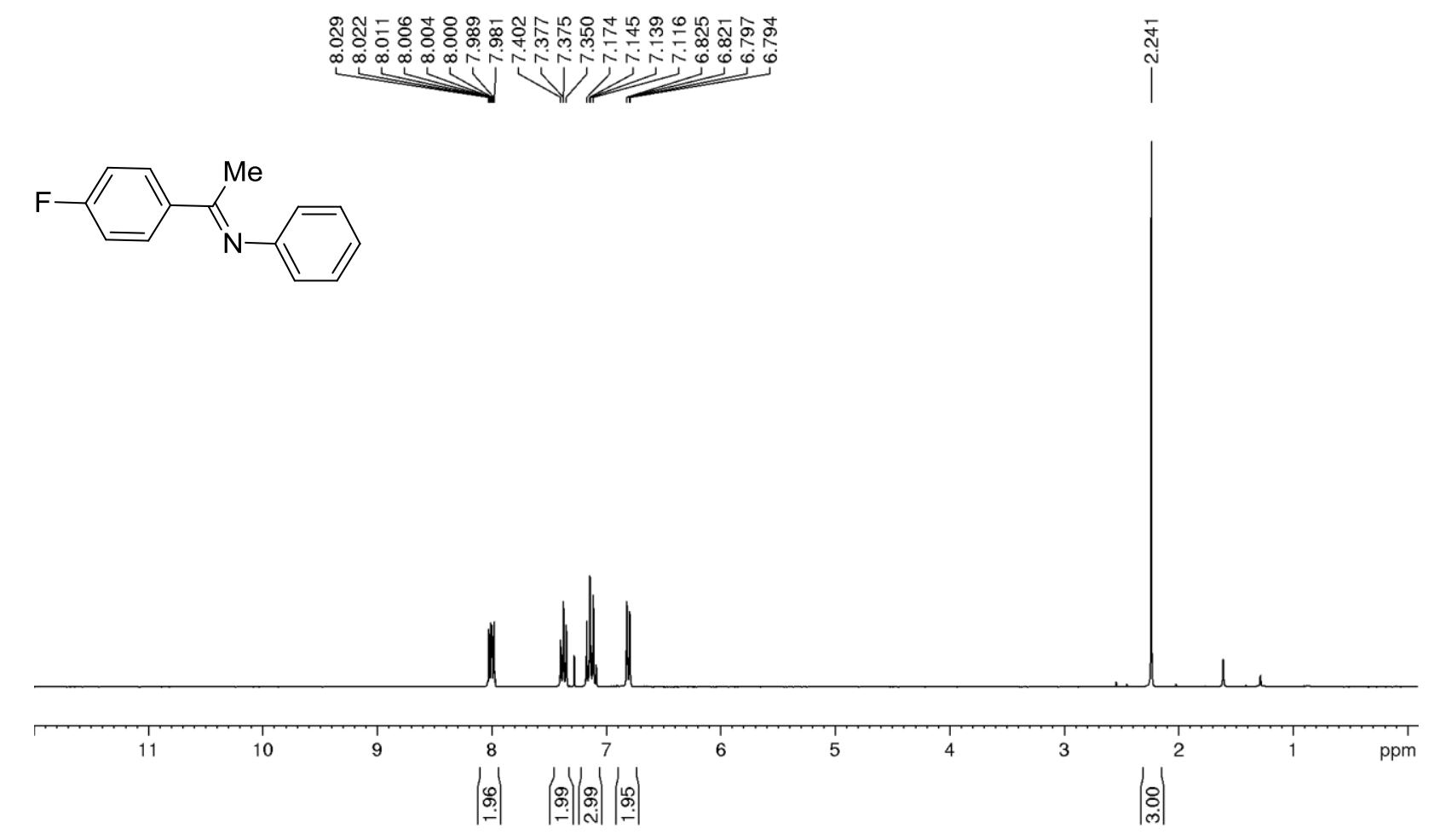

